\title{
Galaxy Clustering in Early Sloan Digital Sky Survey Redshift Data
}

\section{Citation}

Zehavi, Idit, Michael R. Blanton, Joshua A. Frieman, David H. Weinberg, Houjun J. Mo, Michael A. Strauss, Scott F. Anderson, et al. 2002. "Galaxy Clustering in Early Sloan Digital Sky Survey Redshift Data." The Astrophysical Journal 571 (1) (May 20): 172-190. doi:10.1086/339893.

\section{Published Version}

$10.1086 / 339893$

\section{Permanent link}

http://nrs.harvard.edu/urn-3:HUL.InstRepos:33461906

\section{Terms of Use}

This article was downloaded from Harvard University's DASH repository, and is made available under the terms and conditions applicable to Other Posted Material, as set forth at http:// nrs.harvard.edu/urn-3:HUL.InstRepos:dash.current.terms-of-use\#LAA

\section{Share Your Story}

The Harvard community has made this article openly available.

Please share how this access benefits you. Submit a story.

Accessibility 


\title{
GALAXY CLUSTERING IN EARLY SLOAN DIGITAL SKY SURVEY REDSHIFT DATA
}

Idit Zehavi, ${ }^{1,2}$ Michael R. Blanton, ${ }^{1,3}$ Joshua A. Frieman, ${ }^{1,2}$ David H. Weinberg, ${ }^{4}$ Houjun J. Mo, ${ }^{5}$ Michael A. Strauss, ${ }^{6}$ Scott F. Anderson, ${ }^{7}$ James Annis, ${ }^{1}$ Neta A. Bahcall,${ }^{6}$ Mariangela Bernardi, ${ }^{2}$ John W. Briggs, ${ }^{8}$ Jon Brinkmann, ${ }^{9}$ Scott Burles, ${ }^{1}$ Larry Carey, ${ }^{7}$ Francisco J. Castander, ${ }^{2}$ Andrew J. Connolly, ${ }^{10}$ Istvan Csabai, ${ }^{11}, 12$ Julianne J. Dalcanton, ${ }^{7}$ Scott Dodelson,${ }^{1,2}$ Mamoru Doi,,${ }^{13}$ Daniel Eisenstein,,${ }^{14}$ Michael L. Evans, ${ }^{7}$

Douglas P. Finkbeiner, ${ }^{6}$ Scott Friedman, ${ }^{12}$ Masataka Fukugita, ${ }^{15}$ James E. Gunn, ${ }^{6}$ Greg S. Hennessy, ${ }^{16}$

Robert B. Hindsley, ${ }^{17}$ Željko Ivezić, ${ }^{6}$ Stephen Kent,${ }^{1,2}$ Gillian R. Knapp, ${ }^{6}$ Richard Kron,,${ }^{1,2}$ Peter Kunszt, ${ }^{18}$

Donald Q. Lamb,${ }^{2,19}$ R. French Leger, ${ }^{1}$ Daniel C. Long, ${ }^{9}$ Jon Loveday,${ }^{20}$ Robert H. Lupton, ${ }^{6}$ Timothy McKay, ${ }^{21}$ Avery Meiksin, ${ }^{22}$ Aronne Merrelli, ${ }^{23}$ Jeffrey A. Munn, ${ }^{15}$ Vijay Narayanan, ${ }^{6}$ Matt Newcomb,${ }^{24}$ Robert C. Nichol ${ }^{24}$ Russell Owen, ${ }^{7}$ John Peoples, ${ }^{1}$ Adrian Pope, ${ }^{12,24}$ Constance M. Rockosi,${ }^{2}$ David Schlegel, ${ }^{6}$ Donald P. Schneider, ${ }^{25}$ Roman Scoccimarro, ${ }^{3}$ Ravi K. Sheth, ${ }^{1}$ Walter Siegmund, ${ }^{7}$ Stephen Smee, ${ }^{26}$ Yehuda Snir, ${ }^{24}$ Albert Stebbins, ${ }^{1}$ Christopher Stoughton, ${ }^{1}$ Mark SubbaRao, ${ }^{2}$ Alexander S. Szalay, ${ }^{12}$ Istvan Szapudi, ${ }^{27}$ Max Tegmark, ${ }^{28}$ Douglas L. Tucker, ${ }^{1}$ Alan Uomoto, ${ }^{12}$ Dan Vanden Berk, ${ }^{1}$ Michael S. Vogeley, ${ }^{29}$ Patrick Waddell, ${ }^{7}$ BRIAN YANNY, ${ }^{1}$ AND DONALD G. YORK ${ }^{2,19}$ (FOR THE SDSS COLLABORATION)

Received 2001 June 26; accepted 2002 January 29

\begin{abstract}
We present the first measurements of clustering in the Sloan Digital Sky Survey (SDSS) galaxy redshift survey. Our sample consists of 29,300 galaxies with redshifts $5700 \mathrm{~km} \mathrm{~s}^{-1} \leq c z \leq 39,000 \mathrm{~km} \mathrm{~s}^{-1}$, distributed in several long but narrow $\left(2.5-5^{\circ}\right)$ segments, covering $690 \mathrm{deg}^{2}$. For the full, flux-limited sample, the redshiftspace correlation length is approximately $8 h^{-1}$ Mpc. The two-dimensional correlation function $\xi\left(r_{p}, \pi\right)$ shows clear signatures of both the small-scale, "fingers-of-God" distortion caused by velocity dispersions in collapsed objects and the large-scale compression caused by coherent flows, though the latter cannot be measured with high precision in the present sample. The inferred real-space correlation function is well described by a power law, $\xi(r)=\left(r / 6.1 \pm 0.2 h^{-1} \mathrm{Mpc}\right)^{-1.75 \pm 0.03}$, for $0.1 h^{-1} \mathrm{Mpc} \leq r \leq 16 h^{-1} \mathrm{Mpc}$. The galaxy pairwise velocity dispersion is $\sigma_{12} \approx 600 \pm 100 \mathrm{~km} \mathrm{~s}^{-1}$ for projected separations $0.15 \mathrm{~h}^{-1} \mathrm{Mpc} \leq r_{p}$ $\leq 5 h^{-1} \mathrm{Mpc}$. When we divide the sample by color, the red galaxies exhibit a stronger and steeper real-space correlation function and a higher pairwise velocity dispersion than do the blue galaxies. The relative behavior of subsamples defined by high/low profile concentration or high/low surface brightness is qualitatively similar to that of the red/blue subsamples. Our most striking result is a clear measurement of scale-independent luminosity bias at $r \lesssim 10 h^{-1}$ Mpc: subsamples with absolute magnitude ranges centered on $M_{*}-1.5, M_{*}$, and $M_{*}+1.5$ have real-space correlation functions that are parallel power laws of slope $\approx-1.8$ with correlation lengths of approximately 7.4, 6.3, and $4.7 h^{-1} \mathrm{Mpc}$, respectively.
\end{abstract}

Subject headings: cosmology: observations — cosmology: theory — dark matter — galaxies: clusters: general — galaxies: distances and redshifts large-scale structure of universe

\footnotetext{
${ }^{1}$ Fermi National Accelerator Laboratory, P.O. Box 500, Batavia, IL 60510.

2 Astronomy and Astrophysics Department, University of Chicago, Chicago, IL 60637.

${ }^{3}$ Department of Physics, New York University, 4 Washington Place, New York, NY 10003.

${ }^{4}$ Department of Astronomy, Ohio State University, Columbus, OH 43210.

${ }^{5}$ Max-Planck-Institute for Astrophysics, Karl-Schwarzschild-Strasse 1, D-85741 Garching, Germany.

${ }^{6}$ Princeton University Observatory, Peyton Hall, Princeton, NJ 08544.

${ }^{7}$ Department of Astronomy, University of Washington, Box 351580, Seattle, WA 98195.

8 Yerkes Observatory, University of Chicago, 373 West Geneva Street, Williams Bay, WI 53191.

${ }_{9}^{9}$ Apache Point Observatory, P.O. Box 59, Sunspot, NM 88349.

10 University of Pittsburgh, Department of Physics and Astronomy, 3941 O'Hara Street, Pittsburgh, PA 15260.

${ }_{11}$ Department of Physcis, Eötvös University, Budapest, Pf. 32, Hungary, H-1518.

12 Department of Physics and Astronomy, The Johns Hopkins University, 3701 San Martin Drive, Baltimore, MD 21218.

13 Institute of Astronomy and Research Center for the Early Universe, School of Science, University of Tokyo, Tokyo 113-0033, Japan.

${ }^{14}$ Steward Observatory, University of Arizona, 933 North Cherry Avenue, Tucson, AZ 85721.

15 Institute for Cosmic-Ray Research, University of Tokyo, Kashiwa 277-8582, Japan.

${ }^{16}$ US Naval Observatory, 3450 Massachusetts Avenue NW, Washington DC, 20392.

17 Remote Sensing Division, Code 7210, Naval Research Laboratory, 4555 Overlook Avenue SW, Washington, DC 20375.

18 CERN, IT Division, 1211 Geneva 23, Switzerland.

${ }^{19}$ Enrico Fermi Institute, University of Chicago, Chicago, IL 60637.

${ }^{20}$ Sussex Astronomy Centre, University of Sussex, Falmer, Brighton BN1 9QJ, UK

21 Department on Physics, University of Michigan, Ann Arbor, MI 48109.

22 Department of Physics and Astronomy, University of Edinburgh, Blackford Hill, Mayfield Road, Edinburgh, EH9 3JZ, UK

23 Department of Astronomy, California Institute of Technology, Pasadena, CA 91125.

24 Department of Physics, 5000 Forbes Avenue, Carnegie Mellon University, Pittsburgh, PA 15213.

${ }^{25}$ Department of Astronomy and Astrophysics, Pennsylvania State University, University Park, PA 16802.

26 Department of Astronomy, University of Maryland, College Park, MD 20742.

${ }^{27}$ Institute for Astronomy, University of Hawaii, 2680 Woodlawn Drive, Honolulu, HI 96822.

28 Department of Physics, University of Pennsylvania, Philadelphia, PA 19101.

29 Department of Physics, Drexel University, Philadelphia, PA 19104.
} 


\section{INTRODUCTION}

The primary observational goals of the Sloan Digital Sky Survey (SDSS) are to image 10,000 $\mathrm{deg}^{2}$ of the north Galactic cap in five passbands, with an $r$-band limiting magnitude of 22.5, to obtain spectroscopic redshifts of $10^{6}$ galaxies and $10^{5}$ quasars, and to obtain similar data for three $\sim 200 \mathrm{deg}^{2}$ stripes in the south Galactic cap, with repeated imaging to enable co-addition and variability studies in one of these stripes (York et al. 2000). One of the principal scientific objectives is to map the large-scale structure traced by optical galaxies with unprecedented precision over a wide range of scales. These measurements of large-scale structure will allow critical tests of cosmological models and theories of galaxy formation. This paper presents the first measurements of galaxy clustering from the SDSS redshift survey, based on a sample of $\sim 30,000$ galaxies observed during commissioning operations and during the first few months of the survey proper. Complementary studies of the angular clustering of galaxies in the SDSS imaging survey appear in Connolly et al. (2001) and Tegmark et al. (2002), and the implications of these measurements for the three-dimensional galaxy power spectrum are discussed by Dodelson et al. (2002) and Szalay et al. (2001). Scranton et al. (2001) examine many possible systematic effects on the angular clustering measurements and conclude that they are small; these tests and conclusions are also relevant to the analyses of the redshift survey carried out here.

The redshift-space clustering of galaxies has been a central concern of observational cosmology since the early studies of Gregory \& Thompson (1978) and Joeveer \& Einasto (1978). Milestones in this effort include the first CfA redshift survey (Huchra et al. 1983), which mapped 2400 galaxies selected from the Zwicky et al. (1961-1968) catalog over $2.7 \mathrm{sr}$ of sky to a magnitude limit of $m_{\mathrm{Zw}}=14.5$; the Perseus-Pisces survey (Giovanelli \& Haynes 1985) consisting of $\sim 5000$ galaxies chosen from the CGCG and UGC catalogs; redshift surveys in other areas of sky such as the Southern Sky Redshift Survey (da Costa et al. 1991) and the Optical Redshift Survey (Santiago et al. 1995); sparsely sampled surveys of optically selected galaxies to $B \approx 17$ (the Stromlo-APM Redshift Survey: Loveday et al. 1996; the Durham/UKST Redshift Survey: Ratcliffe et al. 1998); the second CfA redshift survey (de Lapparent, Geller, \& Huchra 1986; Geller \& Huchra 1989), with a magnitude limit of $m_{\mathrm{Zw}}=15.5$ and an eventual total of $\sim 13,000$ galaxies in the "Updated Zwicky Catalog" (Falco et al. 1999); a similar extension of the Southern Sky Redshift Survey (da Costa et al. 1998); redshift surveys of IRAS-selected galaxies to successively deeper flux limits of $2 \mathrm{Jy}$ (Strauss et al. 1992), $1.2 \mathrm{Jy}$ (Fisher et al. 1995), and 0.6 Jy (the sparsely sampled QDOT survey: Lawrence et al. 1999; the PSCz survey of $\sim 15,000$ galaxies: Saunders et al. 2001); the deep slice surveys of Vettolani et al. $\left(1998 ; \sim 3300\right.$ galaxies to $\left.b_{J}=19.4\right)$ and Geller et al. (1997; 1800 galaxies to $R=16.13$ ); and the Las Campanas Redshift Survey (LCRS; Shectman et al. 1996), which mapped $\sim 24,000$ galaxies in six thin $\left(1.5 \times 90^{\circ}\right)$ slices at a depth $R \approx 18$. The current state of the art is represented by Peacock et al. (2001) and Percival et al.'s (2001) studies of redshift-space clustering in a sample of $\sim 140,000$ galaxies from the ongoing $2 \mathrm{dF}$ Galaxy Redshift Survey (2dFGRS). The sample that we analyze here is most similar to the LCRS, with slightly more galaxies but a comparable depth and thin-slice geometry.
Two factors that complicate and enrich the interpretation of galaxy clustering in redshift surveys are the distortions of structure induced by peculiar velocities and the possibility that galaxies are "biased" tracers of the underlying matter distribution. On small scales, velocity dispersions in collapsed objects (aka "fingers of God") smear out structures along the line of sight, effectively convolving the real-space correlation function with the galaxy pairwise velocity distribution (see, e.g., Davis \& Peebles 1983). On large scales, coherent flows into high-density regions and out from low-density regions enhance structures along the line of sight (Sargent \& Turner 1977; Kaiser 1987; Regos \& Geller 1991; van de Weygaert \& van Kampen 1993; Hamilton 1998). Because the underlying clustering pattern should be statistically isotropic, the apparent anisotropy induced by redshift-space distortions yields constraints on the distribution of peculiar velocities, which can in turn yield constraints on the matter density parameter $\Omega_{m}$. With our current galaxy sample, we clearly detect the signature of both the small-scale, fingers-of-God suppression and the large-scale, coherent flow amplification. However, we are not yet able to measure the latter effect with high precision, so we defer a detailed examination of $\Omega_{m}$ constraints (and comparison to Peacock et al. 2001) to a future analysis of a larger sample.

The notion that the optical galaxy population might give a systematically " biased" picture of matter clustering came to the fore in the mid-1980s, largely in an effort to reconcile the predictions of $\Omega_{m}=1$ inflationary models with observations (Davis et al. 1985; Bardeen et al. 1986; Melott \& Fry 1986; Bahcall \& Soneira 1983; Kaiser 1984). There are now numerous arguments in favor of a low- $\Omega_{m}$ universe, but theoretical models of galaxy formation, the well-known dependence of observed galaxy clustering on morphological type (e.g., Hubble 1936; Zwicky 1937; Abell 1958; Davis \& Geller 1976; Dressler 1980; Guzzo et al. 1997), and more recent evidence for dependence of clustering on luminosity (e.g., Hamilton 1988; White, Tully, \& Davis 1988; Park et al. 1994; Loveday et al. 1995; Benoist et al. 1998; Willmer, da Costa, \& Pellegrini 1998) all imply that galaxies cannot be perfect tracers of the underlying matter distribution. Advances in hydrodynamic cosmological simulations, highresolution $N$-body simulations, and semianalytic methods now allow detailed a priori predictions of bias for physically motivated models of galaxy formation (e.g., Cen \& Ostriker 1992; Katz, Hernquist, \& Weinberg 1992; Blanton et al. 1999; Colín et al. 1999; Kauffman et al. 1999; Pearce et al. 1999; Benson et al. 2000; White, Hernquist, \& Springel 2001; Yoshikawa et al. 2001). Empirical constraints on bias can therefore provide tests of galaxy formation theories and guidance to physical ingredients that may be missing from current models. The SDSS is ideally suited to the empirical study of bias because of the high sampling density and the detailed photometric and spectroscopic information available for every galaxy. We begin the effort here, by examining the dependence of the real-space correlation function and the redshift-space distortions on galaxy color, luminosity, surface brightness, and light profile concentration.

The next section describes the data sample used for the clustering analysis. Section 3 describes our methods for estimating the correlation function, including technical issues such as sampling corrections and the effects of the minimum fiber spacing in the spectroscopic observations. Section 4 presents the clustering results for the full, flux-limited galaxy 
sample. Section 5 examines the clustering of subsamples defined by color, luminosity, and other galaxy properties. We summarize our results in $\S 6$. A discussion of our jackknife error estimation procedure, and comparison of this procedure to results from mock redshift catalogs, appears in the Appendix.

\section{DATA}

\subsection{Description of the Survey}

The SDSS (York et al. 2000) is producing an imaging and spectroscopic survey over $\pi \mathrm{sr}$ in the north Galactic cap. A dedicated $2.5 \mathrm{~m}$ telescope (W. Siegmund et al. 2002, in preparation) at Apache Point Observatory, Sunspot, New Mexico, images the sky in five bands between 3,000 and 10,000 ( $u, g, r, i, z$; Fukugita et al. 1996) using a drift-scanning, mosaic CCD camera (Gunn et al. 1998), detecting objects to a flux limit of $r \approx 22.5$. Approximately 900,000 galaxies (down to $r_{\text {lim }} \approx 17.77$; Strauss et al. 2002), 100,000 luminous red galaxies (LRGs; Eisenstein et al. 2001), and 100,000 quasars (Richards et al. 2002) are targeted for spectroscopic follow up using two double fiber-fed spectrographs on the same telescope. Most of the essential technical details are summarized in a paper that accompanies the SDSS Early Data Release (C. Stoughton et al. 2002, in preparation).

As of 2001 June, the SDSS has imaged around $2500 \mathrm{deg}^{2}$ of sky and taken spectra of approximately 140,000 objects. We use a subset of these data here to calculate the correlation function of galaxies, confining our attention to regions where the data reductions and calibration have been carefully checked and the spectroscopic completeness is well understood.

\subsection{Imaging and Spectroscopic Pipelines}

As described by C. Stoughton et al. (2002, in preparation), the imaging data are processed by astrometric (J. R. Pier et al. 2002, in preparation) and photometric (Lupton et al. 2001; R. H. Lupton et al. 2002, in preparation) pipelines and calibrated relative to a set of standard stars (Smith et al. 2002). Targets are selected by a target selection pipeline (D. E. Vanden Berk et al. 2002, in preparation), and plates for spectroscopic observations are drilled based on the results of a tiling pipeline (Blanton et al. 2001b). After the spectra are observed, the spectroscopic pipeline then reduces, calibrates, and classifies the spectra and determines redshifts.

The photometric pipeline (R. H. Lupton et al. 2002, in preparation) detects objects and measures their properties in all five bands. Most relevant here are the Petrosian magnitude $m_{P}$, the radius $r_{50}$ containing $50 \%$ of the Petrosian flux, and the radius $r_{90}$ containing $90 \%$ of the Petrosian flux. The details of SDSS Petrosian magnitudes, a modified form of those introduced by Petrosian (1976), are described in a number of references and will not be repeated here, except to say that they are designed to measure a constant (and large) fraction of a galaxy's total light, independent of redshift or central surface brightness but (slightly) dependent on light-profile shape (Blanton et al. 2001a; R. H. Lupton et al., in preparation; Strauss et al. 2002; C. Stoughton et al. 2002 , in preparation; Yasuda et al. 2001). The radii $r_{50}$ and $r_{90}$, which we use below to quantify galaxies' surface brightnesses and morphologies, are not corrected for seeing. However, such corrections would be small since most of the galaxies in this sample are relatively large $\left(r_{50}>2^{\prime \prime}\right.$; Blanton et al. 2001a), and the seeing conditions for the imaging are generally good $\left(\mathrm{FWHM} \lesssim 11^{\prime \prime} 5\right)$.

The flux calibration is performed relative to standard stars as described in York et al. (2000), Smith et al. (2002), and C. Stoughton et al. (2002, in preparation). Calibration is a three-tiered system in which "secondary standards" that are not saturated in the $2.5 \mathrm{~m}$ imaging camera are used to calibrate the imaging data. These secondary standards are themselves calibrated relative to a set of "primary standards" using a $0.5 \mathrm{~m}$ photometric telescope (PT; A. Uomoto et al. 2002, in preparation). These primary standards have been calibrated relative to the fundamental standard $\mathrm{BD}+17^{\circ} 4708$ by the United States Naval Observatory $1 \mathrm{~m}$ telescope. The calibrations used here are not fully validated, though they are thought to be accurate to within $5 \%$. Because of this remaining uncertainty, object magnitudes are referred to in this paper and others based on early SDSS data as $u^{*}, g^{*}, r^{*}, i^{*}$, and $z^{*}$.

The target selection pipeline (D. E. Vanden Berk et al. 2002, in preparation) determines which objects from the imaging survey are spectroscopic targets. We concentrate here on the "main-sample" galaxies in the SDSS, which are selected using the criteria detailed by Strauss et al. (2002). The essential selection criteria for this sample are the stargalaxy separator, the surface brightness limit, and the flux limit. The star-galaxy separation is based on a comparison of the flux of the object measured through a point-spread function aperture to the flux estimated using a best-fit model to the galaxy profile (choosing the better of pure exponential and de Vaucouleurs profiles). This method is known to be an extremely efficient and reliable separator at the magnitudes appropriate for the spectroscopic sample (R. H. Lupton et al. 2002, in preparation). We find that $98 \%$ of objects targeted as main-sample galaxies indeed turn out to be galaxies. The major contaminant is double stars with separations less than $2^{\prime \prime}$.

The surface brightness limit is based on the Petrosian half-light surface brightness in $r^{*}$. For some parts of the sample used here, obtained during commissioning observations, the surface brightness limit is $\mu_{1 / 2}=23.5 \mathrm{mag}$ $\operatorname{arcsec}^{-2}$, but for most of the sample it is $\mu_{1 / 2}=24.5 \mathrm{mag}$ $\operatorname{arcsec}^{-2}$. Because we will use relatively luminous galaxies to trace the density field here, the positive correlation between surface brightness and luminosity (Blanton et al. 2001a) guarantees that the surface brightness limit will be unimportant.

The flux limit of the spectroscopic survey is approximately $r^{*}=17.77$, after correction for Galactic reddening using the maps of Schlegel, Finkbeiner, \& Davis (1998). The limit varies somewhat over the area of our sample, as the target selection criteria changed during the commissioning phase of the survey, when much of these data were taken. We will cut back to a uniform flux limit of $r^{*}=17.6$ for our current analysis. In addition, there is a bright limit imposed on the flux in a $3^{\prime \prime}$ diameter aperture (the entrance aperture of a spectroscopic fiber) of $m_{\text {fiber }}>15$ in $g^{*}, r^{*}$ and $m_{\text {fiber }}>14.5$ in $i^{*}$, in order to avoid saturation and crosstalk between fibers in the spectrograph.

The reliability of the galaxy target selection is very high; galaxy target selection results for two imaging runs over the same patch of sky agree for $95 \%$ of the objects; the differences are attributable to small, random magnitude errors shifting objects across the flux limit (Strauss et al. 2002). 
The tiling pipeline (Blanton et al. 2001b) positions spectroscopic tiles and assigns fibers to targets. The most important constraint is imposed by the size of the fiber plugs, which dictates that two fibers cannot be placed closer than $55^{\prime \prime}$ to each other. If the spectroscopic tiles did not overlap, this would mean that about $10 \%$ of the objects would be unobservable. Because the tiles are circular, about $30 \%$ of the sky is actually covered by more than a single tile; in these regions, many of the objects lost due to collisions of fibers can be recovered. Note, however, that the tiles are positioned such that there are more tiles in dense areas of sky; thus, the regions covered by tile overlaps tend to be $5 \%-10 \%$ overdense compared to average. We will describe in $\S 3$ how we handle objects whose redshifts are missing due to fiber collisions.

Finally, the spectroscopic pipeline extracts, analyzes, and classifies the spectra, determining the spectral type, redshift, and other spectral information for each target. The success rate for classifying spectra and determining redshifts correctly is very high $(>99 \%)$ for main-sample galaxy targets, based on a subsample of $\sim 20,000$ spectra examined by eye. The spectroscopic pipeline assigns an empirically calibrated confidence level to the redshift determination for each object; cutting out main-sample galaxy redshifts with low confidence $(\mathrm{CL}<75 \%)$ removes only $0.7 \%$ of the objects from the sample, with a negligible effect on the clustering results below.

\subsection{Determining Positions, Luminosities, and Rest-Frame Colors}

The redshift of a galaxy is not a linear measure of an object's distance at the moderate redshifts probed here (median $z \sim 0.1$ ), and the comoving distance of an object depends somewhat on the cosmology assumed. Throughout this paper, we assume a Friedmann-Lemaitre metric with $\Omega_{m}=0.3$ and $\Omega_{\Lambda}=0.7$. When we plot correlation functions versus separation, we are always referring to the comoving separation, transformed from $\mathrm{km} \mathrm{s}^{-1}$ separations using the standard formulas as tabulated in, for example, Hogg (1999).

We also must account for cosmological effects when calculating the absolute magnitudes from the apparent magnitude and the redshift using the formula

$$
M=m-\mathrm{DM}(z)-K(z)+5 \log _{10} h,
$$

where $\operatorname{DM}(z)$ is the bolometric distance modulus for the cosmology in question (again, see Hogg 1999), $K(z)$ is the $K$-correction, and the Hubble constant is $H_{0}=100 h \mathrm{~km} \mathrm{~s}^{-1}$ $\mathrm{Mpc}^{-1}$. Throughout this paper, we use $h=1$ to compute absolute magnitudes, and we quote distances in $h^{-1} \mathrm{Mpc}$.

The $K$-correction is necessary to account for the fact that the system response in the observed frame corresponds to a narrower, bluer rest-frame passband, depending on the redshift of the observed object. In order to make an estimate of the $K$-correction, it is therefore necessary to have an estimate of the spectral energy distribution (SED) of each object. We can make a good estimate based on the five-band photometry provided by SDSS. For each object, we find the linear combination of the four SED templates of Coleman, Wu, \& Weedman (1980), as extended in the red and blue by Bolzonella, Miralles, \& Pelló (2000), which best fits the photometry. We use the resulting SED to estimate the $K$ corrections, assuming no evolution of the SED. This method is similar to simply interpolating between passbands to infer a rest-frame flux, while also taking advantage of what astronomers know already about galaxy SEDs. These $K$-corrections are also useful to determine the restframe colors of objects from their observed colors. The details of our procedure, which are based on the photometric redshift methods of Csabai et al. (2000), will be described in a forthcoming paper.

\subsection{Description of the Sample}

Figure 1 shows the angular distribution of the resulting sample in Galactic coordinates. The area covered is approximately $690 \mathrm{deg}^{2}$ (comparable to the sky coverage of the LCRS survey), or about $7 \%$ of the area that will eventually be covered by the survey; in this area, we have selected $\approx 30,000$ galaxies for our sample, as explained in the following paragraphs. Figure 2 shows the distribution in right ascension and redshift of galaxies near the celestial equator $\left(|\delta|<5^{\circ}\right)$.

Even though some regions of the survey are currently complete to $r^{*}<17.77$ (dereddened, using Schlegel et al. 1998), others are complete only to $r^{*}<17.6$, and for simplicity we have pared back our sample to this constant flux limit. In addition, we have imposed a bright limit of $r^{*}>14.5$ because at the bright end we are limited by the bright spectroscopic limits (the $3^{\prime \prime}$ aperture magnitude limit of $r^{*}>15$ imposed to prevent saturation and cross-talk of fibers in the spectrograph) and by the quality of deblending of large galaxies in the version of the photometric pipelines used for targeting many of these galaxies. These flux limits reduce the number of targets we consider by about $10 \%$.

In most of this work, we limit our sample to a fairly small range in redshift, $5,700 \mathrm{~km} \mathrm{~s}^{-1}<c z<39,000 \mathrm{~km} \mathrm{~s}^{-1}$. We do so primarily because it is clear that galaxy evolution within the full range of redshifts (which extends to about $80,000 \mathrm{~km} \mathrm{~s}^{-1}$ ) is important, and at the time of this work there was not yet an adequate model of this evolution to allow proper calculation of the radial selection function. Working at low redshift primarily limits our estimate of the large-scale clustering; however, the thrust of this work is the small-scale clustering of galaxies. Much larger area samples of SDSS galaxies will soon be available, as well as good models of the evolution of the luminosity function, and much better estimates of the large-scale clustering will come from these samples. The outer redshift cut is the most costly of our imposed limits, eliminating $30 \%$ of the objects available after the above flux limits have been imposed.

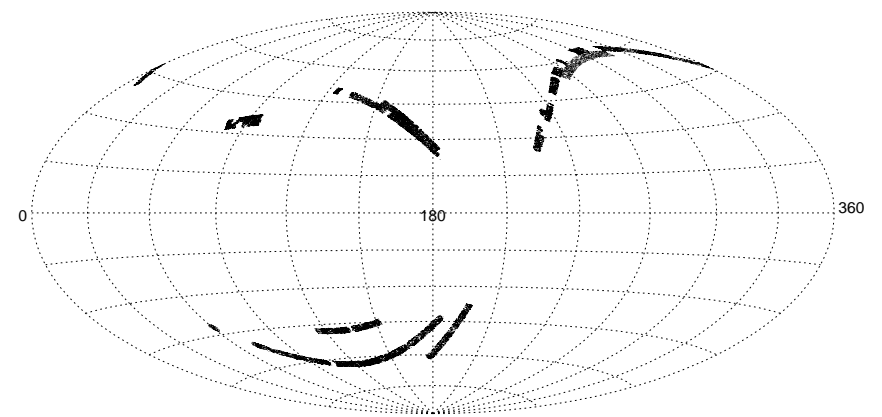

FIG. 1.-Aitoff projection of our galaxy sample in Galactic coordinates. 


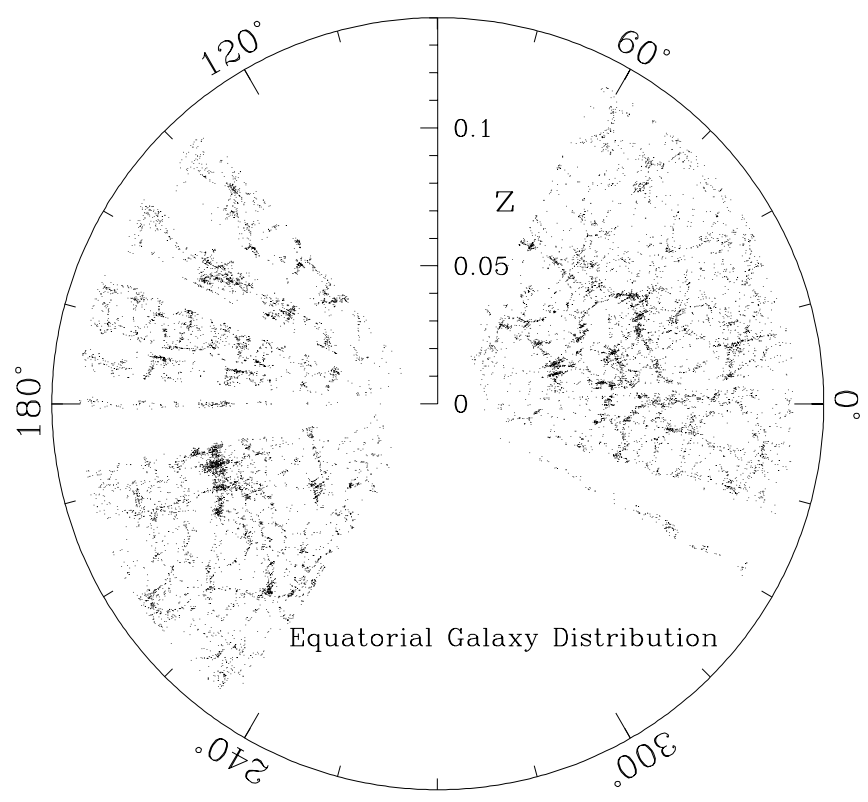

FIG. 2.-Pie-diagram distribution for the equatorial part of our sample. The plot includes 16,300 galaxies that lie within $|\delta|<5^{\circ}$ of the celestial equator.

We wish to study the clustering of relatively luminous galaxies near the exponential cutoff in the luminosity function at $M_{*}$. For most of the work below, we therefore impose absolute magnitude limits of $-22<M_{r} *-5 \log _{10} h<-19$, which roughly brackets the value $M_{*}=-20.8$ determined for the SDSS (Blanton et al. 2001a). These absolute magnitude limits exclude another $15 \%$ of the objects (after the redshift and flux cuts are imposed), leaving us with our canonical sample of 29,300 galaxies. We will use slightly different cuts to define volume limited samples of different luminosity ranges below. Finally, we will compare below the clustering of several different types of galaxies, defined by color, surface brightness, and morphology, describing in the appropriate sections how those subsamples of the canonical sample are defined.

\section{MEASURING THE CORRELATION FUNCTION}

Before measuring the correlation function, we need to determine how to treat the fiber collisions and how to properly correct for angular and radial selection effects. We first detail how we account for these issues, then describe our estimators for the correlation function and its errors.

\subsection{Accounting for Fiber Collisions}

One of the important observational constraints in the SDSS is that no two fibers on the same plate can be closer than $55^{\prime \prime}$. Thus, redshifts for both members of a close galaxy pair can only be obtained in regions where tiles overlap.

If we took no account of fiber collisions at all, then we would systematically underestimate correlations even on large scales because collisions occur more often in overdense regions such as clusters, which have enhanced large-scale clustering for the reasons discussed by, e.g., Kaiser (1984). A simple way to correct this bias is to double-weight the member of each pair that was observed, since its a priori selection probability was $50 \%$. Here we adopt a variant of the double-weighting procedure, assigning each pair member whose redshift was not obtained because of a fiber collision the same redshift as the pair member whose redshift was measured. We term such an assigned redshift a "collision-corrected" redshift. On large scales, where both members of the pair contribute to the same separation bin, the effect is the same as double weighting, but our procedure should perform somewhat better on small scales because it retains information about the known angular positions. Some of the galaxy targets are not assigned fibers due to collisions with quasars or LRGs; in these cases, no redshift is assigned, and the galaxy is treated as if the fiber simply did not measure a redshift successfully, as described in the next subsection.

At $c z=39,000 \mathrm{~km} \mathrm{~s}^{-1}$, the outer edge of our sample, $55^{\prime \prime}$ corresponds to a comoving transverse separation of $0.1 \mathrm{~h}^{-1}$ Mpc. Fiber collisions will have a significant effect on correlation function estimates below this scale, and in this paper we will restrict our measurements to separations greater than $0.1 h^{-1} \mathrm{Mpc}$, so as to avoid the artificial increase of pairs with very small separations. Because two galaxies whose fibers collide also have a line-of-sight separation, the collisions can in principle affect our estimate of the correlation function out to somewhat larger scales, but we show below that these effects are probably smaller than our statistical uncertainties for this sample. In tile overlap regions, which constitute $\sim 30 \%$ of the survey area, we are able to measure redshifts for most of the galaxies which would otherwise be unobtainable due to fiber collisions. We find that roughly $60 \%$ of these galaxies in fact have a redshift within $500 \mathrm{~km} \mathrm{~s}^{-1}$ of their closest angular neighbor. For the rest, either galaxy still has an equal chance of being selected, so double weighting does not statistically bias the correlations with more distant galaxies.

We can use the galaxies in the overlap regions (roughly a third of our full sample) to assess the accuracy of our correction procedure. For the standard analyses in this paper, we use the true redshifts of galaxies whose fibers collide whenever we can obtain them from overlapping tiles and the collision-corrected redshifts from the closest angular neighbor when we cannot. Figure 3 shows the results from the tile overlap regions when we instead use the "collision-corrected" redshifts for all galaxies whose fibers collide. We plot the ratio of the redshift-space correlation function obtained in this way to the one obtained when we use all the available measured redshifts (estimator and errors are defined in 3.4). The collision-corrected redshifts yield a correlation function that is nearly identical to the one obtained from the observed redshifts over a large range of scales. The two results deviate somewhat for small separations, $s<1$ $h^{-1} \mathrm{Mpc}$, but they agree within the statistical uncertainties. Note that these deviations are also partly statistical fluctuations due to the smaller sample used for this comparison. In addition, for the full sample, the fraction of galaxies with collision-corrected redshifts is smaller than the case we examined here, and thus we expect the effects of the fiber collisions to be even smaller.

A detailed examination of fiber collision effects will require tests on artificial catalogs with realistic galaxy clustering and geometry, but the agreement between the dashed line and unity in Figure 3 implies that any residual systematic biases in our correlation function estimates due to fiber collisions are smaller than our current statistical errors. 


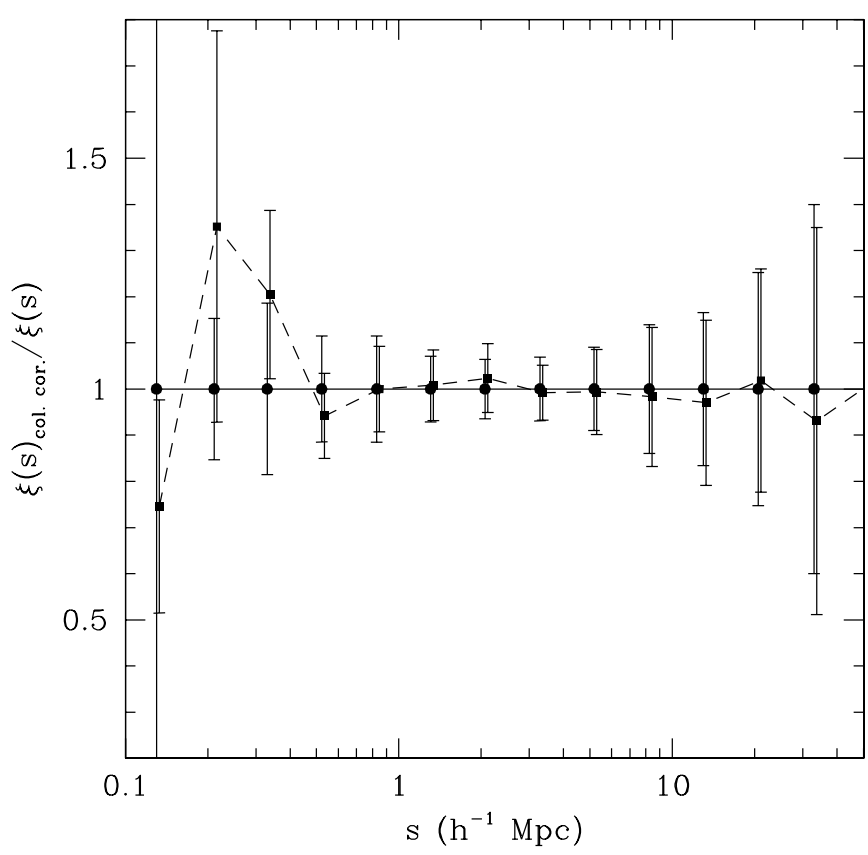

FIG. 3.-Test of the accuracy of our correction for fiber collisions using the tile overlap regions. The dashed line shows the ratio of the redshiftspace correlation function measured using the collision-corrected redshifts for all galaxies whose fibers collide to the result obtained using all the available observed redshifts. The latter estimate (and error bars) is given by the solid line at unity. The points and error bars are shifted slightly for clarity.

\subsection{Angular Selection Function}

A small fraction of the galaxy targets in our sample were not assigned fibers in the observed plates. There are also some galaxy targets whose redshifts are not successfully measured, for the most part because of broken fibers in the spectrograph, but sometimes because of a low signal-tonoise ratio in the spectra. The completeness of the redshift sample, denoted here $f$, thus varies across the survey, and it is necessary to incorporate these variations into the window function of the survey. We evaluate the completeness in the following way. We break up the survey geometry into "sectors" defined by areas of sky covered by unique sets of tiles, as described by Blanton et al. (2001b). For example, if the survey consisted of two tiles, there would be three sectors: the area covered by only the first tile, the area covered by only the second tile, and the overlap area covered by both tiles. These sectors are the natural units in which to divide the survey, and we calculate the completeness $f$ for each sector. The completeness is simply the fraction of objects that were selected as galaxy targets for which a spectral classification was obtained (whether the object turned out to be a galaxy or not), or for which a redshift was assigned because the object was lost in a collision.

In this sample, the average completeness is about $94 \%$. There are two contributions to the incompleteness. First, only $97 \%$ of the available galaxy targets in the regions covered by plates actually are assigned fibers or have a close neighbor that can provide a collision-corrected redshift. This is partly because some galaxies are eliminated due to fiber collisions with quasars, LRGs, or other objects which have higher priority when fibers are assigned, and thus cannot be given collision corrections. In addition, we have included some regions that are covered by two plates, only one of which has so far been observed; the targets in such a region that are assigned to fibers on the unobserved plate contribute to the incompleteness. Second, the fraction of fibers assigned to main galaxy targets that successfully receive classifications and redshifts is about $97 \%$ in this sample. The success rate for obtaining main sample galaxy redshifts during normal survey operations is over $99 \%$. However, some of the data in this sample come from plates that have low signal-to-noise ratio (and will therefore be reobserved later in the survey) or were reduced using older, less efficient versions of the spectroscopic pipeline. In addition, some of these targets are imaging defects that were mistakenly classified as galaxies by early versions of the galaxy target selection algorithm, such as ghost images due to reflections of bright stars inside the camera or satellite trails. Though these latter cases, in fact, do not contribute to galaxy incompleteness, they are included in our estimate of $f$, but this makes a negligible difference to our results.

We apply several masks for regions of particularly bad seeing and where an early version of the tiling algorithm (now replaced) accidentally produced artificial gaps in the sampling. We exclude any objects in our data or random catalogs that lie inside these masks. The masks cover less than $1 \%$ of the total area. We have not applied masks around bright stars; if we did, they would exclude about $1 \%$ of the total area (Scranton et al. 2001). It will be necessary to include these masks when studying clustering at the largest scales, because at large scales the clustering amplitude of stars becomes large (due to the variation with Galactic latitude) and the clustering amplitude of galaxies becomes small.

We properly take into account the incompleteness in each individual sector when calculating the correlation function. But, in fact, because the completeness of the redshift sample is high to begin with, the effects of completeness variations on our current clustering measurements are negligible. We have verified this by calculating the correlation function, not accounting for the incompleteness, obtaining almost indistinguishable results.

\subsection{Radial Selection Function}

As noted above, our sample is limited at bright and faint apparent magnitudes: $14.5<r^{*}<17.6$. Thus, at any given redshift we can only observe galaxies in a given absolute magnitude range. Furthermore, we restrict our analysis here to galaxies with absolute magnitudes $-22<M_{r^{*}}<-19$. At any redshift, the fraction of objects in this absolute magnitude range that are in the sample is

$$
\phi(z)=\frac{\int_{M_{\min }(z)}^{M_{\max }(z)} d M \Phi(M)}{\int_{-22}^{-19} d M \Phi(M)},
$$

where $\Phi(M)$ is the luminosity function (number density of objects per unit magnitude) and

$$
\begin{aligned}
& M_{\min }(z)=\max [-22,14.5-\mathrm{DM}(z)-K(z)], \\
& M_{\max }(z)=\min [-19,17.6-\mathrm{DM}(z)-K(z)],
\end{aligned}
$$

and $\operatorname{DM}(z)=m-M$ is the distance modulus as described in $\S 2.3$. In this context, $K(z)$ is determined using the mean galaxy SED in the sample. Equations (2), (3) and (4) simply express the fact that a galaxy must lie in our apparent mag- 
nitude range and in our absolute magnitude range to be included in the sample.

The luminosity function for our sample is determined in the manner described by Blanton et al. (2001a). It is necessary to perform this calculation separately for each of the subsamples described in $\S 5$ because the luminosity functions of, for example, blue galaxies and red galaxies differ substantially. The luminosity function for our full sample is consistent with that of Blanton et al. (2001a) when determined using the same redshift limits as that paper. However, we note here that it appears from preliminary results (to be described in detail elsewhere) that the galaxy luminosity function evolves measurably within the redshift range of our spectroscopic sample. At the time the calculations presented here were performed, we had not yet accounted for this effect in our calculation of the selection function. This is our main motivation for limiting the current sample to $c z<39,000 \mathrm{~km} \mathrm{~s}^{-1}$. More recently, we have fitted a pure luminosity evolution model to the data. The resulting change in the selection function below $c z=39,000 \mathrm{~km} \mathrm{~s}^{-1}$ is less than $5 \%$, and the resulting differences in the measured correlation functions are negligible. Thus we are confident that our radial selection function calculated without accounting for evolution is sufficient to study the small-scale clustering of interest here.

When the random sample is created for the calculation of the correlation function (see below), this selection function $\phi(z)$ and the local completeness $f$ must be taken into account. In practice, we first distribute points uniformly in comoving space; we then include each such point in the random sample with a probability $f \phi(z)$. Figure 4 compares the expected redshift distribution of this uniform sample (smooth line) to the actual redshift histogram of galaxies, including the galaxies whose redshifts were assigned by our collision correction method. The differences between the expected redshift distribution and the actual one reflect the large-scale structure that we are here attempting to measure.

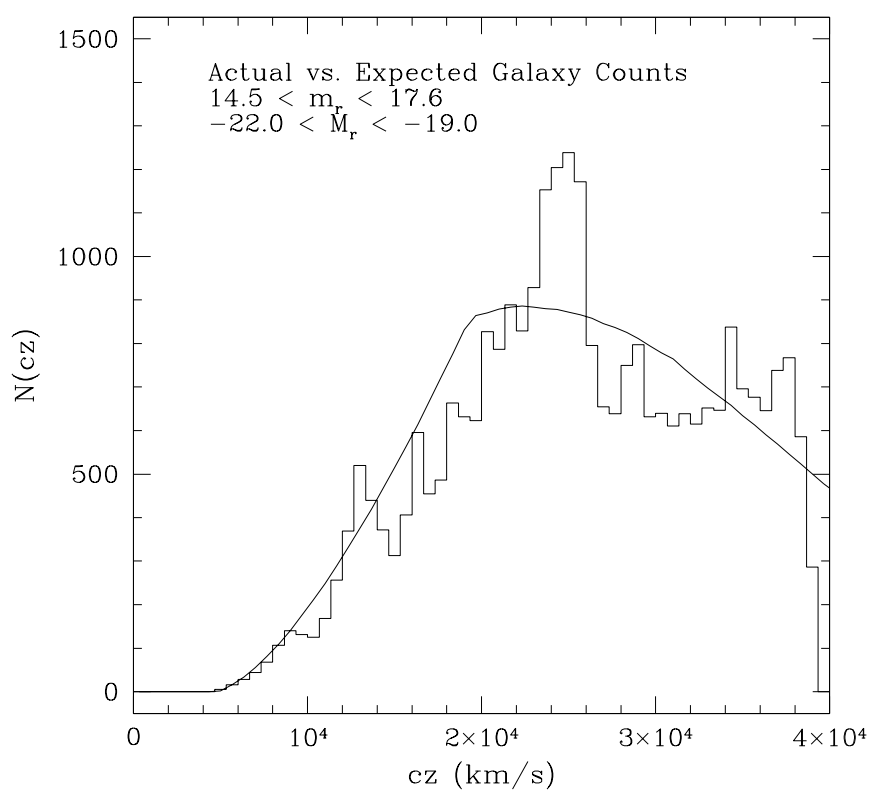

FIG. 4.- Histogram of the redshift distribution of the SDSS galaxies in our sample. The solid line is the average distribution expected given the luminosity function, the flux limits, and the angular selection function.

\subsection{Estimator}

To account for the survey geometry, we generate random catalogs of galaxies with the same survey geometry as the real sample, applying both the radial and angular selection functions. We typically use in each random catalog 10 times the number of galaxies in the real sample, and we have verified that further increasing the number of random points makes negligible difference to the results.

We calculate the correlation function using the Landy \& Szalay (1993) estimator,

$$
\xi(s)=\frac{1}{N_{\mathrm{RR}}(s)}\left[N_{\mathrm{DD}}(s)\left(\frac{n_{R}}{n_{D}}\right)^{2}-2 N_{\mathrm{DR}}(s) \frac{n_{R}}{n_{D}}+N_{\mathrm{RR}}(s)\right],
$$

where $N_{\mathrm{DD}}, N_{\mathrm{DR}}$, and $N_{\mathrm{RR}}$ are the weighted data-data, data-random, and random-random pair counts, respectively, with redshift-space separations in a bin centered on $s$, and $n_{D}$, and $n_{R}$ are the mean number densities of galaxies in the data and random samples. Bins in $s$ are logarithmically spaced with width of 0.2 in $\log \left(s / h^{-1} \mathrm{Mpc}\right)$ starting from $s=0.1 h^{-1}$ Mpc. Other statistics are calculated in an analogous way. We also tried the alternative estimators of Davis $\&$ Peebles (1983) and Hamilton (1993) and found no significant difference in the results.

For the pair weighting we follow Hamilton (1993) and use a minimum variance weighting (see also Davis \& Huchra 1982; Feldman, Kaiser, \& Peacock 1994). For a galaxy pair with redshift separation $s$, we weight each galaxy by

$$
w_{i}=\frac{1}{1+4 \pi n_{D} f_{i} \phi\left(z_{i}\right) J_{3}(s)},
$$

where $J_{3}(s) \equiv \int_{0}^{s} s^{\prime 2} \xi\left(s^{\prime}\right) d s^{\prime}$. For this integral, we approximate $\xi$ by a power law with slope -1.2 and correlation length $8 h^{-1} \mathrm{Mpc}$ (resembling the result for the correlation function in redshift space that we get below, see $\S 4.1$ ), but the results are robust to reasonable choices. Alternatively, we also weighted each galaxy simply by the inverse of the (radial and angular) selection function and obtained comparable results.

The full covariance error matrices are obtained by a jackknife error estimate (see, e.g., Lupton 1993). We divide our sample into 10 separate regions on the sky of approximately equal area. We perform the analysis 10 times, each time leaving a different region out. The estimated statistical covariance of $\xi_{i}$ in redshift separation bin $i$ and $\xi_{j}$ in $\operatorname{bin} j$ is then

$$
\operatorname{Covar}\left(\xi_{i}, \xi_{j}\right)=\frac{N-1}{N} \sum_{l=1}^{N}\left(\xi_{i}^{l}-\bar{\xi}_{i}^{l}\right)\left(\xi_{j}^{l}-\bar{\xi}_{j}^{l}\right)
$$

where $N=10$ in our case and $\bar{\xi}_{i}$ is the mean value of $\xi_{i}$ measured in the samples. Further discussion regarding the robustness of the jackknife error estimate and comparison to alternative error estimates can be found in the Appendix. Note that if the number of regions is increased $(N>10)$, then each term in the sum decreases (because the $N-1$ regions in each jackknife subsample are a larger fraction of the total sample), but the number of terms increases, so the estimated covariance converges to a stable answer.

In what follows, we present results for the Landy-Szalay estimator, with minimum variance weighting for the gal- 
axies, and errors obtained by jackknife resampling. Galaxies with missing redshifts due to fiber collisions are accounted for as described above in $\S 3.1$.

\section{CLUSTERING OF THE FULL SAMPLE}

In this section we present the results for our full galaxy sample. Summarizing the details described in the previous sections, the sample consists of 29,300 galaxies with redshift $5,700 \mathrm{~km} \mathrm{~s}^{-1}<c z<39,000 \mathrm{~km} \mathrm{~s}^{-1}$, apparent magnitude (corrected for Galactic absorption) $14.5<r^{*}<17.6$, and absolute magnitude $-22<M_{r^{*}}<-19$.

\subsection{Redshift-Space Clustering}

Figure 5 shows the redshift-space correlation function $\xi(s)$ of the full sample. For separations $2 h^{-1} \mathrm{Mpc}<s<10$ $h^{-1} \mathrm{Mpc}$, the observed correlation function can be crudely approximated by a power law, $\xi(s)=\left(s / s_{0}\right)^{-\gamma}$, with $\gamma_{s}=1.2$ and $s_{0}=8.0 h^{-1}$ Mpc. Table 1 summarizes our results for the full sample together with the corresponding results obtained for some other major redshift surveys available in the literature. In our comparison to other surveys, we focus largely on the LCRS (Shectman et al. 1996), as this survey resembles ours most closely in terms of selection, geometry, and analysis. Their results for the redshift-space correlation function are shown as well in Figure 5 (open squares; taken from Tucker et al. 1997). The LCRS results are in quite good agreement with ours, though the SDSS correlation function has a slightly higher amplitude. We have assumed an $\Omega_{m}=0.3, \Omega_{\Lambda}=0.7$ model to compute comoving separations, but adopting an Einstein-de Sitter model (EdS; as Tucker et al. do) yields a nearly indistinguishable result. The SDSS $\xi(s)$ remains measurably nonzero out to $s=30 h^{-1} \mathrm{Mpc}$ and is consistent with zero at larger separations.

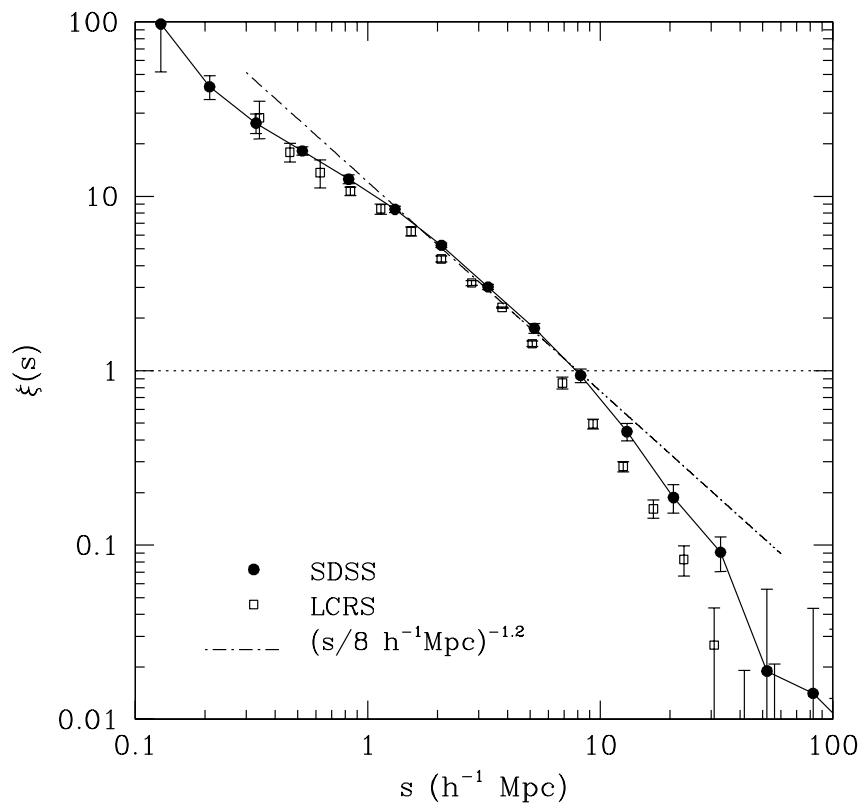

FIG. 5.-Redshift-space correlation function $\xi(s)$ ( filled circles, solid line). The error bars plotted here and in all subsequent figures correspond to the $1 \sigma$ uncertainty estimated from jackknife resampling. A fiducial power-law fit for the range $2 h^{-1} \mathrm{Mpc}<s<10 h^{-1} \mathrm{Mpc}$ is plotted as a dot-dashed line. Open squares show $\xi(s)$ obtained from the LCRS (Tucker et al. 1997).
The redshift-space correlation function $\xi(s)$ differs from the real-space correlation function $\xi_{r}(r)$ because of peculiar velocities. Following standard practice, we separate the effects of redshift-space distortions from spatial correlations by separating the vector between two galaxies into a line-ofsight component $\pi$ and a projected component $r_{p}$, and measuring $\xi\left(r_{p}, \pi\right)$. More specifically, following the notation of Fisher et al. (1994), for a pair of galaxies with redshift positions $\boldsymbol{v}_{1}$ and $\boldsymbol{v}_{2}$, we define the redshift separation vector $\boldsymbol{s} \equiv \boldsymbol{v}_{1}-\boldsymbol{v}_{2}$ and the line-of-sight vector $1 \equiv \frac{1}{2}\left(\boldsymbol{v}_{1}+\boldsymbol{v}_{2}\right)$. This allows us to define the parallel and perpendicular separations

$$
\pi \equiv \boldsymbol{s} \cdot 1 /|1|, \quad r_{p}^{2} \equiv \boldsymbol{s} \cdot \boldsymbol{s}-\pi^{2} .
$$

In real space, the contours of equal $\xi$ should be circular (by isotropy, $\xi$ depends only on the scalar separation), but in redshift space the contours are distorted by peculiar velocities.

Figure 6 shows $\xi\left(r_{p}, \pi\right)$ for our sample, where we bin $r_{p}$ and $\pi$ in linear bins of $2 h^{-1} \mathrm{Mpc}$. On small scales, the contours are elongated along the line of sight direction, exhibiting the expected fingers-of-God distortion caused by velocity dispersion in collapsed objects. On larger scales, $\xi\left(r_{p}, \pi\right)$ shows compression in the $\pi$ direction, caused by coherent large-scale streaming. The qualitative appearance of Figure 6 is similar to that of, e.g., Figure 1 of Fisher et al. (1994) or Figure 2 of Peacock et al. (2001).

\subsection{Real-Space Clustering}

The effects of redshift-space distortions are only radial, so projection onto the $r_{p}$ axis gives information about the realspace correlation function. We compute the projected corre-

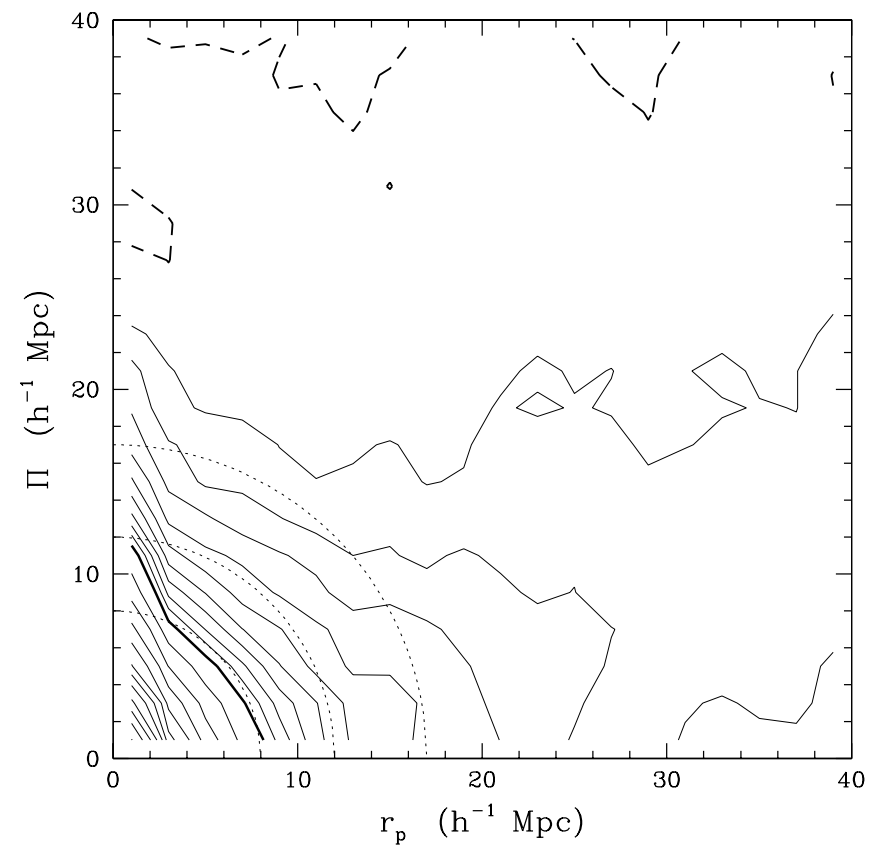

FIG. 6.-Contours of $\xi\left(r_{p}, \pi\right)$, the correlation function as a function of separation perpendicular $\left(r_{p}\right)$ and parallel $(\pi)$ to the line of sight. The heavy solid contour corresponds to $\xi=1$; for larger values of $\xi$ contours are logarithmically spaced, with $\Delta \log _{10} \xi=0.1$; below $\xi=1$ they are linearly spaced, with $\Delta \xi=0.1$; the heavy dashed contour corresponds to $\xi=0$. The concentric dotted lines are the angle-averaged redshift-space correlation function, $\xi(s)$, at $\xi(s)=1.0,0.5$, and 0.25 
TABLE 1

Clustering Results of Different Galaxy Redshift Surveys

\begin{tabular}{ccccccc}
\hline \hline Survey & $N_{\text {gal }}$ & $s_{0}$ & $\gamma_{s}$ & $r_{0}$ & $\gamma$ & $\left(1 h^{-1} \mathrm{Mpc}\right)$ \\
\hline SDSS $^{\mathrm{a}} \ldots \ldots .$. & 29,300 & $\sim 8.0$ & $\sim 1.2$ & $6.14 \pm 0.18$ & $1.75 \pm 0.03$ & $640 \pm 60$ \\
$2 \mathrm{dF}^{\mathrm{b}} \ldots \ldots \ldots . .$. & $15,123^{\mathrm{c}}$ & $\ldots$ & $\ldots$ & $4.92 \pm 0.27$ & $1.71 \pm 0.06$ & $\ldots$ \\
$\mathrm{LCRS}^{\mathrm{d}} \ldots \ldots$. & 26,400 & $6.3 \pm 0.3$ & $1.52 \pm 0.03$ & $5.06 \pm 0.12$ & $1.86 \pm 0.03$ & $570 \pm 80$ \\
PSCz $^{\mathrm{e}} \ldots \ldots .$. & 15,400 & 5.0 & 1.2 & 3.7 & 1.69 & $350 \pm 60$ \\
$\mathrm{CfA2}^{\mathrm{f}} \ldots \ldots .$. & 12,800 & $\sim 7.5$ & $\sim 1.6$ & 5.8 & 1.8 & $540 \pm 180$ \\
ORS $^{\mathrm{g}} \ldots \ldots .$. & 8,500 & $7.6 \pm 1.2$ & $1.6 \pm 0.1$ & $6.1 \pm 1.2$ & $1.6 \pm 0.1$ & $\ldots$ \\
\hline
\end{tabular}

Note.-Values of $s_{0}$ and $r_{0}$ are in units of $h^{-1} \mathrm{Mpc}, \sigma_{12}$ is in units of $\mathrm{km} \mathrm{s}^{-1}$.

a We use comoving distances assuming $\Omega_{m}=0.3 \Omega_{\Lambda}=0.7$. With an Einstein-de Sitter model we get $r_{0}=5.7 \pm 0.2$ and $\sigma_{12}\left(1 h^{-1} \mathrm{Mpc}\right)=590 \pm 50$. Note that a power law is a poor fit to $\xi(s)$, though a good fit to $\xi_{r}(r)$.

${ }^{b}$ Norberg et al. 2001; these are the fit parameters for a volume-limited sample of galaxies with $-19.5<M_{b_{J}}<-20$, close to $M^{*}=-19.7$ (Folkes et al. 1999).

${ }^{c}$ Here 15,123 refers to a volume-limited sample, drawn from a flux-limited sample containing $\sim 160,000$ galaxies.

d Tucker et al. 1997; Jing et al. 1998 (both assuming an EdS model).

e Jing et al. 2002, using 9400 galaxies (EdS cosmology). As galaxies are selected from the IRAS cata$\log$, they are preferentially late types, and thus are more directly comparable to our "blue" galaxies sample, see $\S 5.1$.

f Values of $s_{0}$ and $\gamma_{s}$ are taken from de Lapparent, Geller, \& Huchra 1988, using 1,800 galaxies of first slice; $r_{0}$ and $\gamma$ are based on Fig. 3 of Marzke et al.'s 1995 analysis of CfA2 and SSRS2; $\sigma_{12}$ from Marzke et al. 1995.

g Hermit et al. 1996.

lation function $w_{p}\left(r_{p}\right)$ by integrating $\xi\left(r_{p}, \pi\right)$ over $\pi$,

$w_{p}\left(r_{p}\right) \equiv 2 \int_{0}^{\infty} d \pi \xi\left(r_{p}, \pi\right)=2 \int_{0}^{\infty} d y \xi_{r}\left(\sqrt{r_{p}^{2}+y^{2}}\right)$,

where $\xi_{r}$ is the desired real-space correlation function (Davis $\&$ Peebles 1983). In practice we integrate up to $\pi_{\max }=40$ $h^{-1} \mathrm{Mpc}$, which is large enough to include most correlated pairs and to give a stable result. The second equation (righthand side) above allows us to relate $w_{p}$ to the real-space correlation function. In particular, for a power law $\xi_{r}(r)=\left(r / r_{0}\right)^{-\gamma}$, the second integral can be done analytically, yielding

$$
\begin{aligned}
& w_{p}\left(r_{p}\right)=A r_{p}^{1-\gamma} \\
& \quad \text { with } A=r_{0}^{\gamma} \Gamma(0.5) \Gamma[0.5(\gamma-1)] / \Gamma(0.5 \gamma),
\end{aligned}
$$

where $\Gamma$ is the Gamma function.

Figure 7 shows $w_{p}\left(r_{p}\right)$ for the full galaxy sample and the best-fit power-law model, which corresponds to $\xi_{r}(r)=\left(r / r_{0}\right)^{-\gamma} \quad$ with $\quad r_{0}=6.14 \pm 0.18 \quad h^{-1} \quad \mathrm{Mpc}$ and $\gamma=1.75 \pm 0.03$. This fit to the slope and amplitude of the correlation function is obtained using points in the range $0.1 h^{-1} \mathrm{Mpc}<r_{p}<16 h^{-1} \mathrm{Mpc}$; the correlation coefficient between $r_{0}$ and $\gamma$, measuring the normalized covariance of the two estimates, is $\sim-0.5$, implying that the measures are anticorrelated to a degree. Since the jackknife estimates of the off-diagonal terms in the covariance matrix are noisy and lead to an unstable matrix inversion in the $\chi^{2}$ minimization (unless we confine the fit to only a few bins), the best-fit $r_{0}$ and $\gamma$ values were obtained from the diagonal terms only. As a result, we are not guaranteed to have unbiased estimates of these parameters, but the visually evident goodness of fit suggests that any such bias is negligible. The errors on $r_{0}$ and $\gamma$ were obtained from the variance in the estimates of these quantities among the jackknife subsamples, again using only the diagonal terms in the covariance matrix, as described in the Appendix.
The real-space correlation function is characterized much more accurately by a power law than the redshift-space correlation function. Our value of $\gamma$ agrees well with results from previous redshift surveys and angular clustering studies (e.g., Davis \& Peebles 1983; Loveday et al. 1995; Table 1) and with the slope derived from the SDSS angular correlation function (Connolly et al. 2001). The value of $r_{0}$ is also similar to that obtained from other optically selected galaxy samples, as can be seen in Table 1, though in some cases

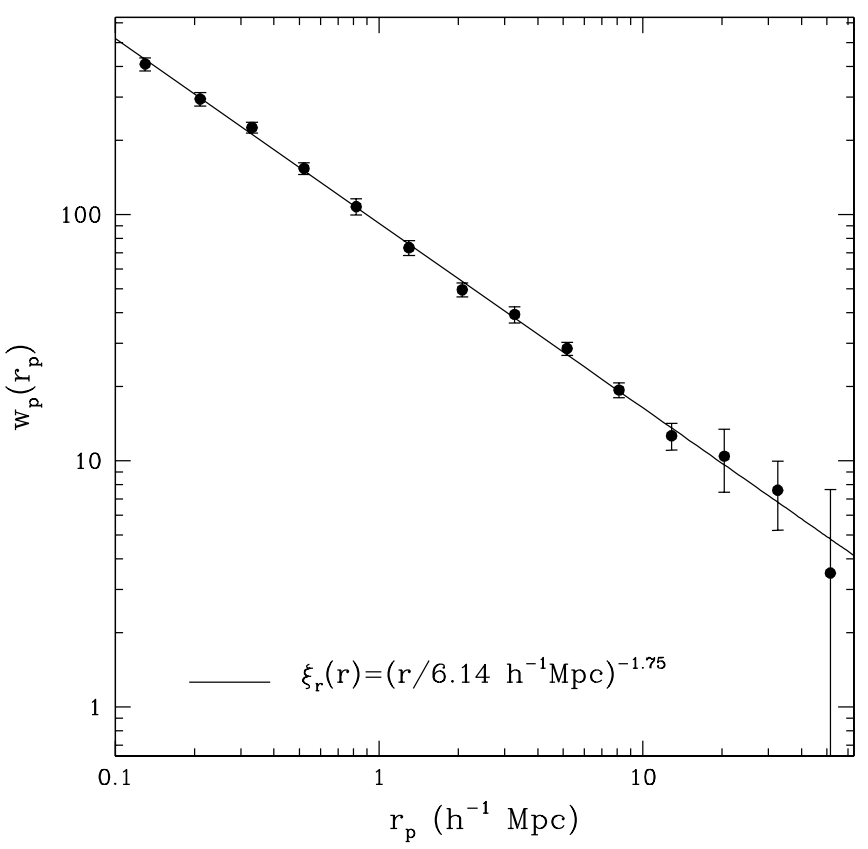

FIG. 7.-Projected correlation function $w_{p}\left(r_{p}\right)$ (filled circles). The solid line is the best-fit power-law for $w_{p}$, which implies the denoted power-law for the real-space correlation function $\xi_{r}(r)$. The fit is performed for $r_{p}<16 h^{-1} \mathrm{Mpc}$ 
slightly on the high side; for example, Jing, Mo, \& Börner (1998) find $r_{0}=5.06 \pm 0.12 h^{-1} \mathrm{Mpc}$ for the LCRS. If we adopt an EdS cosmology, as they do, instead of a flat- $\Lambda$ cosmology, then our inferred value of $r_{0}$ drops slightly, to $r_{0}=5.7 \pm 0.2 h^{-1} \mathrm{Mpc}$. Remaining differences may be attributed to the specifics of the selection criteria and magnitude ranges, reflecting the dependence of galaxy clustering on color and luminosity. Our value of $r_{0}$ is also higher than the value $r_{0}=4.92 \pm 0.27$ found for $b_{J}$-selected galaxies with $M \sim M_{*}$ in the $2 \mathrm{dFGRS}$ by Norberg et al. (2001), but it is similar to the value they obtain for galaxies $0.5-1 \mathrm{mag}$ brighter than $M *($ see their Table 1$)$.

\subsection{Angular Moments}

The redshift-space correlation function $\xi(s)$ in Figure 5 differs from the real-space correlation function $\xi_{r}(r)$ inferred from $w_{p}\left(r_{p}\right)$ in the expected sense: $\xi(s)$ is depressed on small scales by velocity dispersions and enhanced on large scales by coherent flows, so the slope of $\xi(s)$ is shallower and $s_{0}>r_{0}$. The anisotropy of $\xi\left(r_{p}, \pi\right)$ encodes more complete information about the amplitude of galaxy peculiar velocities. In principle, the anisotropy on large scales can be used to constrain $\beta \equiv \Omega_{m}^{0.6} / b$, where the bias parameter $b$ is the ratio of galaxy fluctuations to mass fluctuations (Kaiser 1987; Hamilton 1992). For this application, it is helpful to decompose $\xi\left(r_{p}, \pi\right)$ into a sum of Legendre polynomials,

$$
\xi\left(r_{p}, \pi\right)=\sum_{l} \xi_{l}(s) \mathscr{P}_{l}(\mu),
$$

where $\mathscr{P}_{l}$ is the $l$ th Legendre polynomial and $\mu$ is the cosine of the angle between the line of sight and the redshift separation vector. The angular moments are found by integration

$$
\xi_{l}(s)=\frac{2 l+1}{2} \int_{-1}^{1} \xi\left(r_{p}, \pi\right) \mathscr{P}_{l}(\mu) d \mu .
$$

In linear perturbation theory, only the monopole, $\xi_{0}(s)$, quadrupole, $\xi_{2}(s)$, and hexadecapole, $\xi_{4}(s)$ are nonzero, and the ratio

$$
\begin{aligned}
Q(s) & \equiv \frac{\xi_{2}(s)}{\left(3 / s^{2}\right) \int_{0}^{s} \xi_{0}\left(s^{\prime}\right) s^{\prime 2} d s^{\prime}-\xi_{0}(s)}=G(\beta) \\
& \equiv \frac{(4 / 3) \beta+(4 / 7) \beta^{2}}{1+(2 / 3) \beta+(1 / 5) \beta^{2}}
\end{aligned}
$$

Hamilton (1992). Thus, the ratio $Q$ provides an estimate of $\beta$ (similar estimates can be constructed using $\xi_{4}(s)$, but they are noisier). However, while linear theory distortions produce a negative quadrupole term, fingers-of-God distortions produce a positive quadrupole, and their signature persists out to large separations (Cole, Fisher, \& Weinberg 1994; Fisher et al. 1994).

Figure 8 shows the quadrupole ratio $Q(s)$ for the full sample. The error bars are obtained, as before, from the scatter in the jackknife subsamples. This figure quantifies the visual impression of the contours in Figure 6, showing positive (fingers-of-God) quadrupole distortion at $s \lesssim 10 h^{-1} \mathrm{Mpc}$ and negative (coherent flow) quadrupole distortion at larger scales. $Q(s)$ should approach a constant value in the linear regime, and the measured results are consistent with this prediction. However, the error bars on these scales are large and highly correlated, whereas high precision over a range of scales is needed to separate the influence of coherent flows

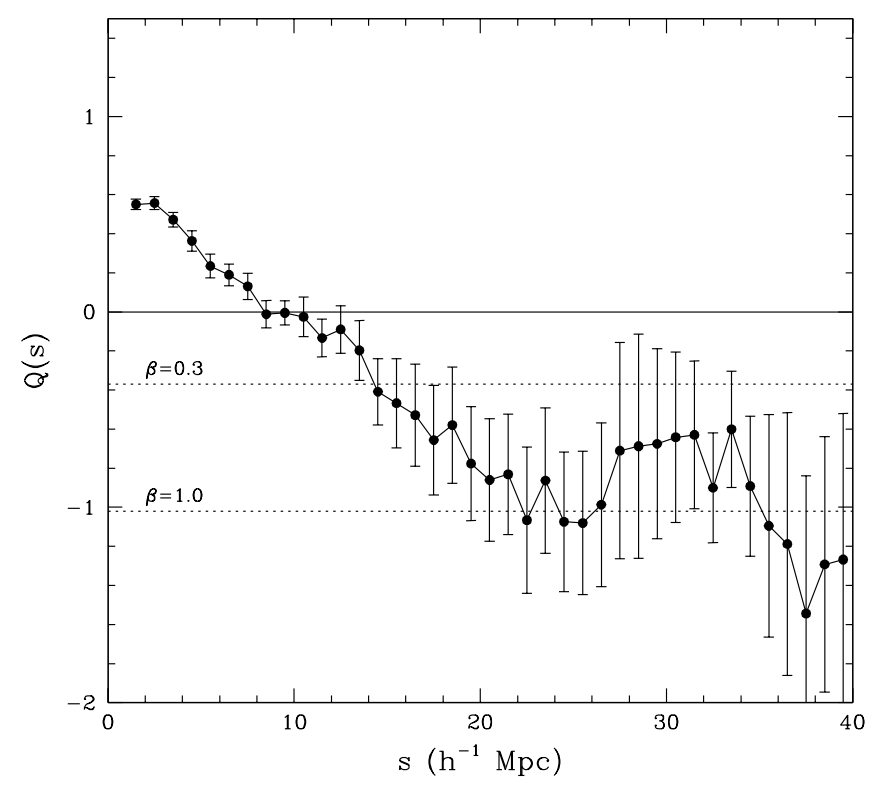

FIG. 8.-Modified quadrupole to monopole ratio, $Q=\xi_{2} /\left(\bar{\xi}_{0}-\xi_{0}\right)$. In linear theory this ratio is determined by the parameter $\beta \equiv \Omega_{m}^{0.6} / b$. Dotted lines show the linear theory expectation for $\beta=0.3$ and $\beta=1.0$.

from that of small-scale dispersions (see, e.g., Hatton \& Cole 1998). The effective volume of our current sample is $\sim 4 \times 10^{6}\left(h^{-1} \mathrm{Mpc}\right)^{3}$. Our measurement of large-scale redshift-space distortions is limited by finite volume effects, as a small number of elongated superclusters and filaments in the data can give rise to anisotropy in $\xi\left(r_{p}, \pi\right)$ on large scales. In this respect, the thin-slice geometry of our present sample works against us, since it provides relatively few pairs at large transverse separations. We therefore defer an estimate of $\beta$ to a future study based on a larger, more nearly threedimensional sample of SDSS data, and focus instead on the amplitude of small-scale, incoherent velocities (but see Peacock et al. 2001 for an estimate of $\beta$ from the 2 dFGRS survey using a similar statistic).

\subsection{Pairwise Velocity Dispersion}

In the nonlinear regime, where density and velocity fields are weakly coupled, the correlation function $\xi\left(r_{p}, \pi\right)$ can be modeled as a convolution of $\xi_{r}(r)$ with the galaxy pairwise velocity distribution $F(V)$ (Peebles 1980, § 76; Davis \& Peebles 1983; see Fisher 1995 for an illuminating discussion of the assumptions implicit in this approach). If $F$ varies only slowly with $r$, one can write

$1+\xi\left(r_{p}, \pi\right)=H_{0} \int_{-\infty}^{\infty}\left[1+\xi_{r}\left(\sqrt{r_{p}^{2}+y^{2}}\right)\right] F(V) d y$,

where

$$
V \equiv \pi-H_{0} y+\bar{v}_{12}(r)
$$

and $\bar{v}_{12}(r)$ is the mean radial pairwise velocity of galaxies at separation $r$. The real-space correlation function $\xi_{r}(r)$ can be inferred from $w_{p}\left(r_{p}\right)$, as described in $\S 4.2$. Unfortunately, the forms of $\bar{v}_{12}(r)$ and $F(V)$ are not known a priori for galaxies. Following Davis \& Peebles (1983), we assume that 
$\bar{v}_{12}(r)$ in the above equation takes the form

$$
\bar{v}_{12}(r)=\frac{H_{0} y}{1+\left(r / r_{0}\right)^{2}} .
$$

This model is based on the similarity solution of the pair conservation equation (Davis \& Peebles 1977). Using the formulae given in Mo, Jing, \& Börner (1997), it can be shown that equation (16) with $r_{0} \sim 5 h^{-1}$ Mpc matches reasonably well the mean streaming velocities of dark matter particles in the $\Lambda$ CDM model with $\Omega_{m}=0.3$ and $\sigma_{8} \sim 1$. The similarity solution may therefore be a reasonable approximation for the underlying density field over limited ranges of length and time scales. If galaxies are biased relative to the mass with a constant bias factor independent of time, the mean streaming velocities for galaxies should have a similar form (see Fisher et al. 1994). Our following presentation is based on equation (16), but we will test the sensitivity of our results to this assumed infall model.

Based on observational (Davis \& Peebles 1983; Fisher et al. 1994; Marzke et al. 1995) and theoretical (e.g., Peebles 1976; Diaferio \& Geller 1996; Sheth 1996; Juszkiewicz, Fisher, \& Szapudi 1998) considerations, we adopt an exponential form for $F$,

$$
F(V)=C \exp \left(-2^{1 / 2}|V| / \sigma_{12}\right),
$$

where $C$ is a normalization factor and $\sigma_{12}(r)$ is the pairwise velocity dispersion (PVD). Under these assumptions, we can estimate $\sigma_{12}(r)$ by performing a $\chi^{2}$ minimization of the difference between the observed $\xi\left(r_{p}, \pi\right)$ and the prediction given by equation (14). In practice, we minimize the following quantity,

$$
\sum_{i}\left[\frac{\xi^{\mathrm{obs}}\left(r_{p}, \pi_{i}\right)-\xi^{\mathrm{pred}}\left(r_{p}, \pi_{i} ; \sigma_{12}\right)}{\sigma_{\xi}^{\mathrm{obs}}\left(r_{p}, \pi_{i}\right)}\right]^{2},
$$

where the summation is done over $\pi$ bins up to $15 h^{-1} \mathrm{Mpc}$ for a fixed $r_{p}$, so generally $\sigma_{12}$ is a function of $r_{p}$. Here $\sigma_{\xi}^{\text {obs }}\left(r_{p}, \pi\right)$ is the error on $\xi\left(r_{p}, \pi\right)$ estimated from the jackknife samples. The fit for $\sigma_{12}$ is robust to changing the limiting $\pi$ in the range $10-20 h^{-1} \mathrm{Mpc}$.

Figure 9 shows the result of this calculation, the PVD of the full sample for projected separations $0.1 h^{-1}$ Mpc $<r_{p}<20 h^{-1} \mathrm{Mpc}$. The error bars are obtained by fitting $\sigma_{12}$ separately from each of the jackknife samples and computing the associated jackknife error (analogous to the way we obtain errors on the power-law fit for $w_{p}$ ). This provides a realistic estimate of the errors, which are dominated by variations in the number of rare, high-dispersion structures in the sample (see discussions by Mo et al. 1993; Zurek et al. 1994; Marzke et al. 1995; Somerville, Davis, \& Primack 1997). Figure 10 compares the function $\xi\left(r_{p}, \pi\right)$ predicted by the best-fit model to the measured values for several different choices of $r_{p}$.

As a test of the sensitivity of our results to the assumed form of $\bar{v}_{12}$, we have repeated the analysis where $\bar{v}_{12}$ is assumed to be the same as that for dark matter particles in the $\Lambda$ CDM model (calculated using the formulae in Mo et al. 1997). This assumption would be valid if the mean streaming velocity of galaxy pairs at a given separation is the same as that of mass particles at the same separation. We find that for $r_{p} \lesssim 3 h^{-1} \mathrm{Mpc}$, the PVD is quite similar to that obtained assuming the similarity model (eq. [16]), while

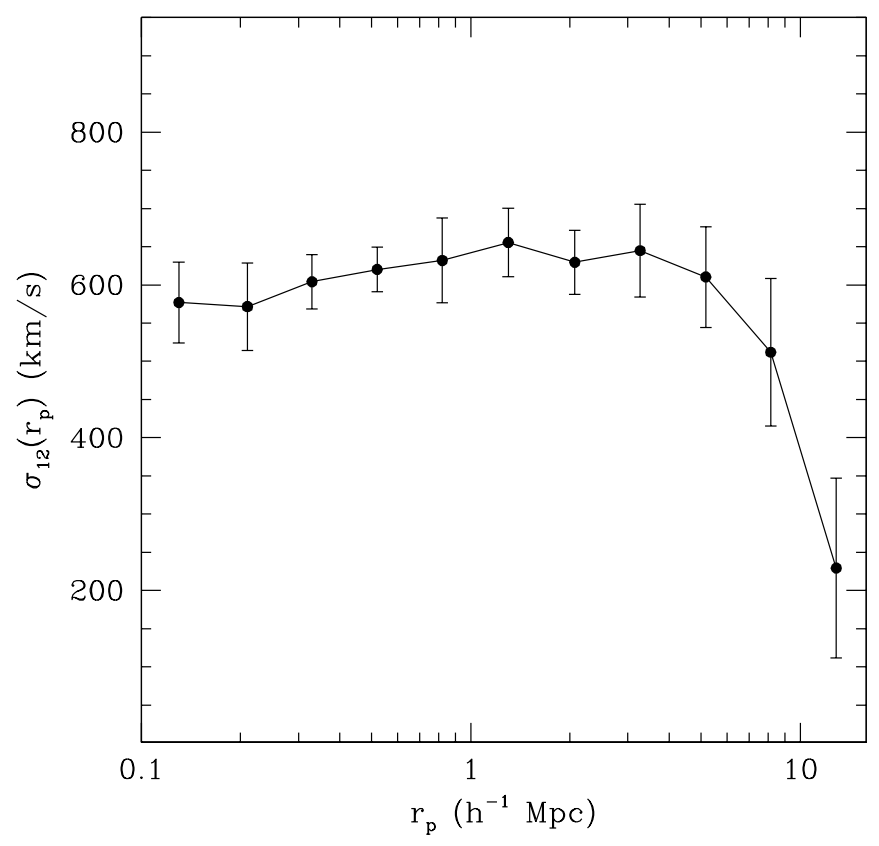

FIG. 9.-Pairwise velocity dispersion $\sigma_{12}\left(r_{p}\right)$, inferred by fitting $\xi\left(r_{p}, \pi\right)$. Error bars are obtained from the values of $\sigma_{12}$ in different jackknife subsamples. The value of $\sigma_{12}$ at $r_{p}>3 h^{-1} \mathrm{Mpc}$ depends significantly on the assumed mean streaming model.

at larger separations it changes significantly. Without knowing how galaxies are biased relative to the mass, it is unclear which infall model is more realistic. The test we describe here, however, indicates that estimates of the PVD at $r_{p} \lesssim 3 h^{-1} \mathrm{Mpc}$ are robust to uncertainties in the infall model.

The measured PVD is roughly constant in this range, with $\sigma_{12}(r) \simeq 550-675 \mathrm{~km} \mathrm{~s}^{-1}$. If we adopt an EdS cosmology,

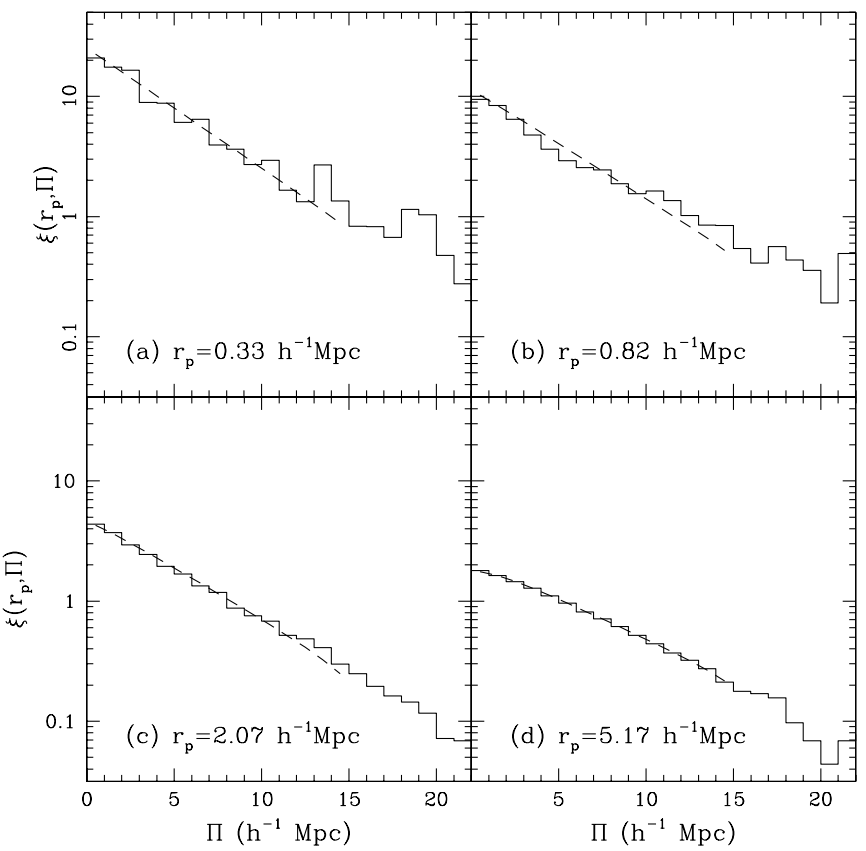

FIG. 10.-Examples of the model fits for $\xi\left(r_{p}, \pi\right)$ for four different values of $r_{p}$. The histogram shows the observed values, and the dashed line is the model fit of eq. (14). 
$\sigma_{12}$ decreases by $\approx 50-100 \mathrm{~km} \mathrm{~s}^{-1}$. The last column in Table 1 presents the values of $\sigma_{12}$ at $r_{p}=1 h^{-1}$ Mpc obtained by our analysis and some other redshift surveys. Our estimate is close to the values found by Jing et al. (1998) for the LCRS, $570 \mathrm{~km} \mathrm{~s}^{-1}$, and by Marzke et al. (1995) for CfA2+SSRS2, $540 \mathrm{~km} \mathrm{~s}^{-1}$, but it is substantially higher than the values found in the early 1980s from much smaller redshift surveys $\left(250 \mathrm{~km} \mathrm{~s}^{-1}\right.$ by Bean et al. $1983 ; 340 \mathrm{~km} \mathrm{~s}^{-1}$ by Davis \& Peebles 1983). The SDSS result thus confirms that the galaxy velocity field, while colder than predicted by unbiased $\Omega_{m}=1$ models (e.g., Davis et al. 1985), is not so cold that it demands an extremely low value of $\Omega_{m}$ or a highly biased galaxy distribution. While $\sigma_{12}(r)$ has been the most widely used characterization of small-scale velocity dispersions, other statistics have been proposed that are less sensitive to rare objects that contribute many pairs (Davis, Miller, \& White 1997; Landy, Szalay, \& Broadhurst 1998) or that quantify the dispersion as a function of local density (Strauss, Ostriker, \& Cen 1998). Future measurements that examine the dispersion as a function of both environment and type may prove a valuable diagnostic for the relation between galaxies and dark matter halos (Sheth et al. 2001b).

\section{DEPENDENCE ON GALAXY PROPERTIES}

The SDSS is ideal for investigating the dependence of clustering on galaxy properties because a wealth of photometric data is available for each galaxy in the spectroscopic sample. Here we examine the dependence of the real-space correlation function and redshift-space anisotropy on galaxy color, then calculate the real-space correlation function for subsamples defined by luminosity, surface brightness, and light-profile concentration. The spirit of our investigation is similar to that of Guzzo et al.'s (1997) study of galaxy clustering as a function of morphological type and luminosity, but the higher quality of our imaging data allows us to consider a broader set of photometric parameters, and the larger size of our redshift sample allows us to measure differences in clustering with higher precision.

\subsection{Color}

We divide our full sample into two subsamples based on the rest-frame $u^{*}-r^{*}$ colors of the galaxies. Strateva et al. (2001) find that the $u^{*}-r^{*}$ color distribution of galaxies is bimodal, and thus galaxies can be naturally divided into "blue" and "red" classes. They also show (using independent morphological classification schemes) that the blue class contains mainly late (spiral) morphological types while the red class consists mainly of bulge-dominated galaxies, as one would expect. After $K$-corrections are accounted for, we find that the color distribution is still bimodal but that the division at $u^{*}-r^{*}=2.2$ in observed bands is closer to $u^{*}-r^{*}=1.8$ in rest-frame bands. We therefore divide the sample into galaxies bluer and redder than a rest-frame color of $u^{*}-r^{*}=1.8$, resulting in a red subsample that includes $\sim 20,000$ galaxies and a blue subsample of $\sim 10,000$ galaxies. In the full absolute magnitude range considered here $\left(-19<M_{r^{*}}<-22\right)$, the two subsamples have similar space densities, but the red galaxies are systematically more luminous (Blanton et al. 2001a) and therefore sample a larger volume. We list some relevant properties of the full sample and the color subsamples in the first lines of Table 2. Space densities $\bar{n}$ are the inferred mean density for the indicated class of galaxies over the full absolute magnitude range. We also repeated our clustering analysis defining the blue and red samples based on the rest-frame $g^{*}-r^{*}$ color (making the division at $g^{*}-r^{*}=0.6$ ), and found very similar results.

Figure 11 compares the redshift-space correlation functions of the red and blue subsamples to that of the full galaxy sample. The red galaxies have a substantially higher amplitude and steeper $\xi(s)$ than the blue galaxies, with a correlation length $s_{0} \approx 9 \mathrm{~h}^{-1} \mathrm{Mpc}$ compared to $s_{0} \approx 5.5 \mathrm{~h}^{-1}$ $\mathrm{Mpc}$ for the blue galaxies. This difference is expected from the well-known morphology-density relation (Dressler 1980), since redder, early-type galaxies preferentially inhabit high-density regions. The difference in anisotropy of $\xi\left(r_{p}, \pi\right)$ is equally striking (Fig. 12), with red galaxies exhibiting much stronger fingers-of-God distortions on small scales. The compression of contours along the $\pi$ axis at large scales is also much more obvious for the red galaxies, though this may be just a consequence of the smaller number and weaker clustering of the blue galaxies, which makes $\xi\left(r_{p}, \pi\right)$ much noisier.

Because the peculiar velocity distortions are very different for the two subsamples, it is important to remove them in order to assess their relative spatial clustering. Figure 13 compares the projected correlation functions $w_{p}\left(r_{p}\right)$, with the red galaxies again exhibiting a steeper and higher amplitude correlation function. Power-law model fits in the range $0.1 h^{-1} \mathrm{Mpc}<r_{p}<16 h^{-1} \mathrm{Mpc}$ yield $r_{0}=6.78$ $\pm 0.23 h^{-1} \mathrm{Mpc}, \gamma=1.86 \pm 0.03$ for the red galaxies and $r_{0}=4.02 \pm 0.25 h^{-1} \mathrm{Mpc}, \gamma=1.41 \pm 0.04$ for the blue galaxies. The blue galaxies show hints of a departure from power-law behavior at the smallest separations, a possible

TABLE 2

FluX-Limited CORRELATion Function SAMPles

\begin{tabular}{|c|c|c|c|c|c|c|}
\hline Description & Additional limits & $N_{\text {gal }}$ & $\bar{n}$ & $r_{0}$ & $\gamma$ & $r_{r_{0} \gamma}$ \\
\hline Full.. & & 29,300 & 1.85 & $6.14 \pm 0.18$ & $1.75 \pm 0.03$ & -0.51 \\
\hline Red.. & $u-r>1.8$ & 19,603 & 1.05 & $6.78 \pm 0.23$ & $1.86 \pm 0.03$ & -0.15 \\
\hline 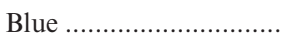 & $u-r<1.8$ & 9,532 & 0.87 & $4.02 \pm 0.25$ & $1.41 \pm 0.04$ & -0.24 \\
\hline 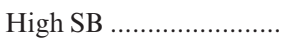 & $\mu_{1 / 2, r^{*}}<20.5$ & 17,859 & 0.94 & $6.48 \pm 0.21$ & $1.84 \pm 0.03$ & -0.14 \\
\hline 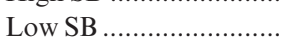 & $\mu_{1 / 2, r^{*}} * 20.5$ & 11,439 & 0.98 & $5.55 \pm 0.21$ & $1.55 \pm 0.04$ & -0.47 \\
\hline High concentration ....... & $c=r_{90} / r_{50}>2.7$ & 11,883 & 0.55 & $6.74 \pm 0.24$ & $1.88 \pm 0.02$ & -0.29 \\
\hline Low concentration ........ & $c=r_{90} / r_{50}<2.7$ & 17,417 & 1.41 & $5.64 \pm 0.22$ & $1.63 \pm 0.03$ & -0.01 \\
\hline
\end{tabular}

Note.-All samples use $14.5<r^{*}<17.6,5,700 \mathrm{~km} \mathrm{~s}^{-1}<c z<39,000 \mathrm{~km} \mathrm{~s}^{-1}$ and $-22<M_{r} *-19 ; \bar{n}$ is measured in units of $10^{-2} h^{3} \mathrm{Mpc}^{-3} ; r_{0}$ is in units of $h^{-1} \mathrm{Mpc} ; r_{0}$ and $\gamma$ are obtained from a fit for $w_{p}\left(r_{p}\right)$; and $r_{r_{0} \gamma} \equiv \sigma_{r_{0} \gamma} /\left(\sigma_{r_{0}} \sigma_{\gamma}\right)^{1 / 2}$ is the correlation coefficient between $r_{0}$ and $\gamma$. 


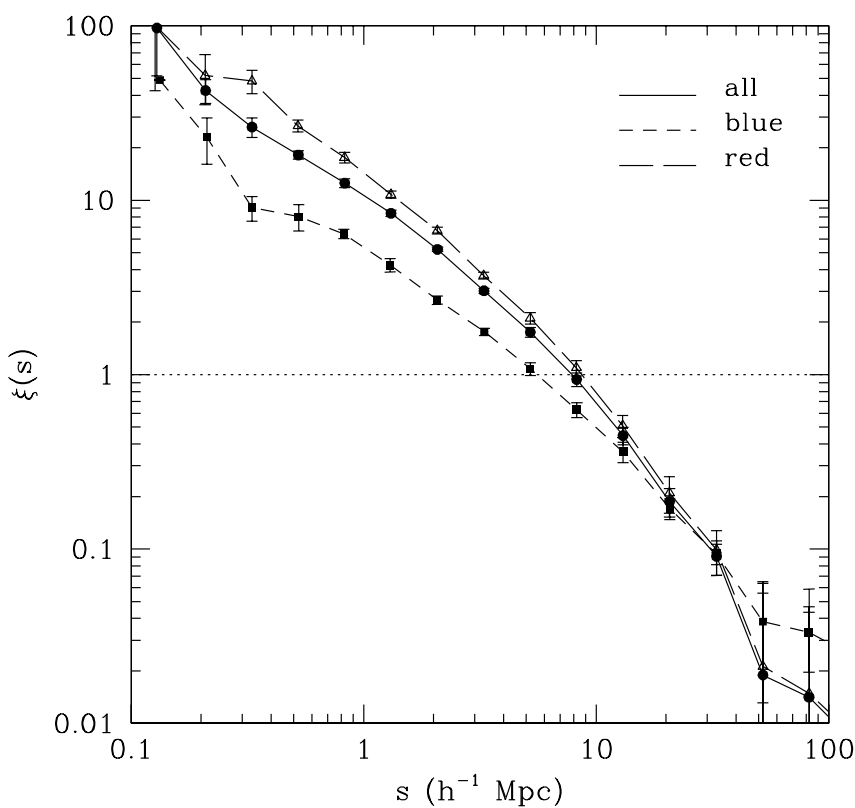

FIG. 11.-Redshift-space correlation function $\xi(s)$ for the blue sample (filled squares, short-dashed line), the red sample (open triangles, longdashed line), and the full sample (filled circles, solid line). Color cut is based on $u^{*}-r^{*}$ color.

signature of their tendency to cluster in lower mass halos with smaller virial radii (see, e.g., Seljak 2000), but the statistical significance of this departure is not high. At large scales, the two correlation functions approach each other, with $w_{p}\left(r_{p}\right)$ for the red galaxies having a slightly higher amplitude but similar shape. The behavior in Figure 13 is qualitatively consistent with expectations based on the morphology-density relation (Narayanan, Berlind, \& Weinberg 2000, Fig. 2), though the data at large scales are too noisy to test whether the relative bias becomes constant in the linear regime, as "local" bias models predict (Coles 1993; Fry \& Gaztañaga 1993; Mann, Peacock, \& Heavens 1998; Scherrer \& Weinberg 1998; Narayanan et al. 2000).

Figure 14, the quadrupole ratio $Q(s)$, confirms the much stronger fingers-of-God distortion of the red galaxies evident in Figure 12, with a large positive $Q$ at small scales. At large scales, the red galaxies have a more negative $Q(s)$ than the blue galaxies, which is contrary to expectation given their higher relative bias, but the difference is marginal at best; for $s>15 h^{-1} \mathrm{Mpc}$, both subsamples generally have $Q(s)$ within the $1 \sigma$ error bar of the full sample $Q(s)$. With
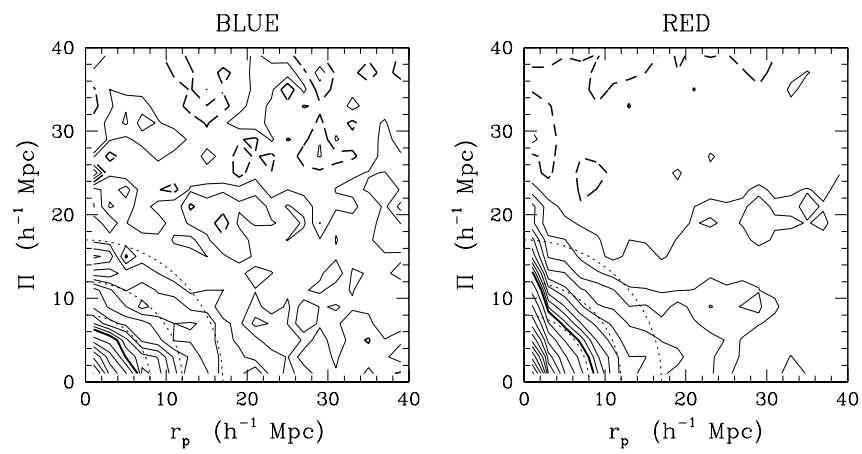

FIG. 12.- $\xi\left(r_{p}, \pi\right)$ for the (left $)$ blue sample and (right) red sample. Contours are as in Fig. 6.

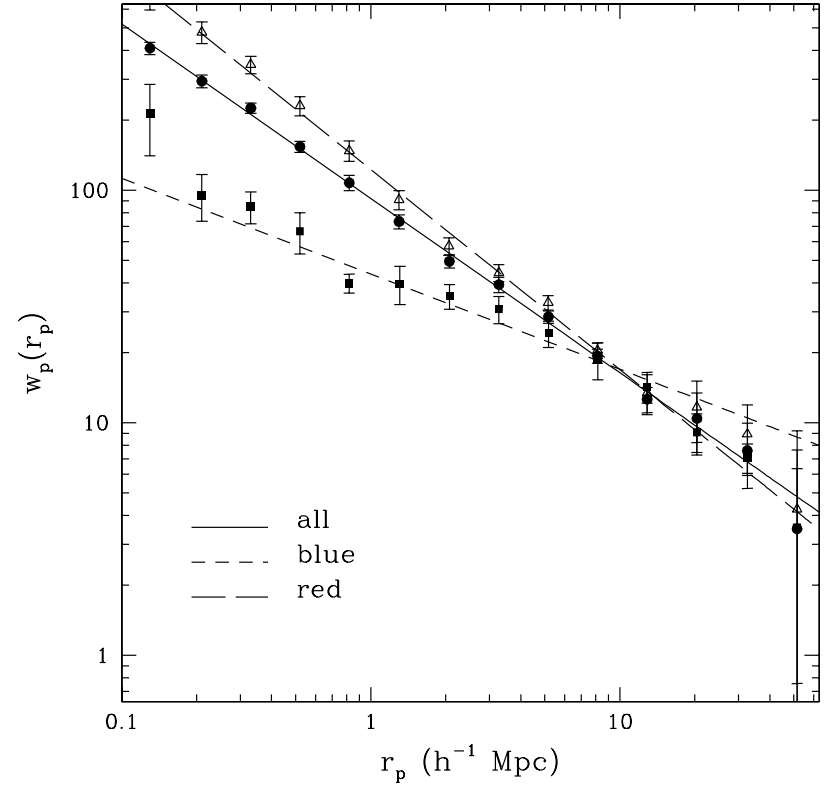

FIG. 13.-Projected correlation functions $w_{p}\left(r_{p}\right)$ for the blue (squares), red (triangles), and full (circles) samples. The straight lines are the best-fit power-laws for $w_{p}$, obtained for $0.1 h^{-1} \mathrm{Mpc}<r_{p}<16 h^{-1} \mathrm{Mpc}$. The short-dashed, long-dashed, and solid lines correspond to the blue, red, and full samples, respectively.

future, larger samples, comparison of real-space clustering amplitudes and redshift-space distortions on large scales will allow interesting new tests of bias models.

Figure 15 shows the PVDs of the two subsamples, demonstrating very clearly the preference of red galaxies for denser, hotter environments. For $r_{p} \sim 0.2-8 h^{-1} \mathrm{Mpc}$, the PVD of the red sample is $\sigma_{12} \approx 650-750 \mathrm{~km} \mathrm{~s}^{-1}$, while the blue galaxy PVD is only $\sigma_{12} \approx 300-450 \mathrm{~km} \mathrm{~s}^{-1}$. This latter range is in fact similar to that obtained by Fisher et al. (1994) for IRAS galaxies. The amplitude and scale-depend-

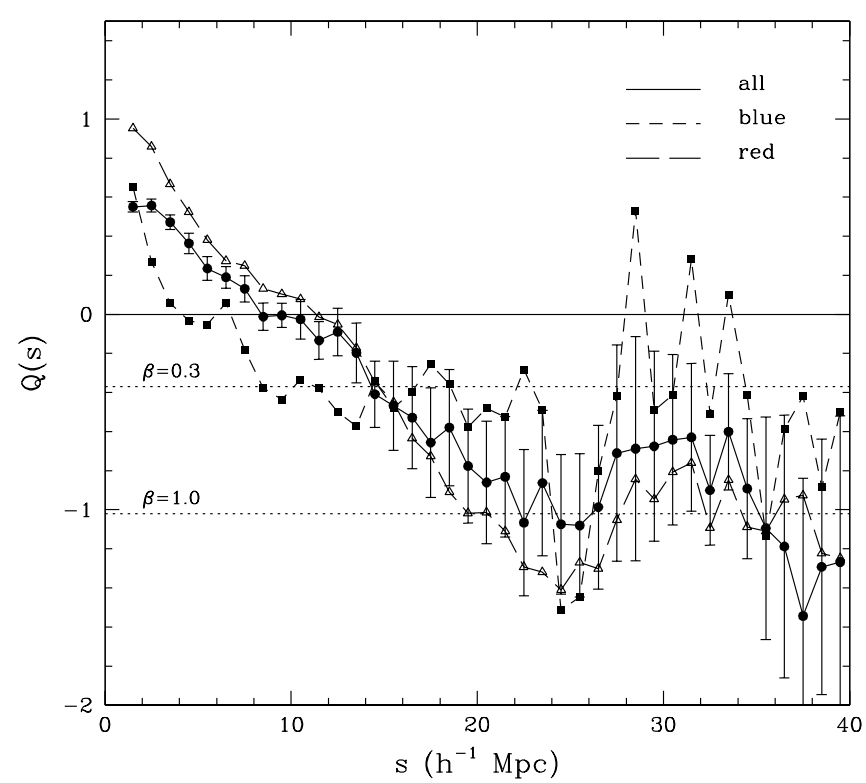

FIG. 14. $-Q(s)$ for the blue (short-dashed line), red (long-dashed line), and full (solid line) samples. For clarity, error bars are drawn only for the full sample. 


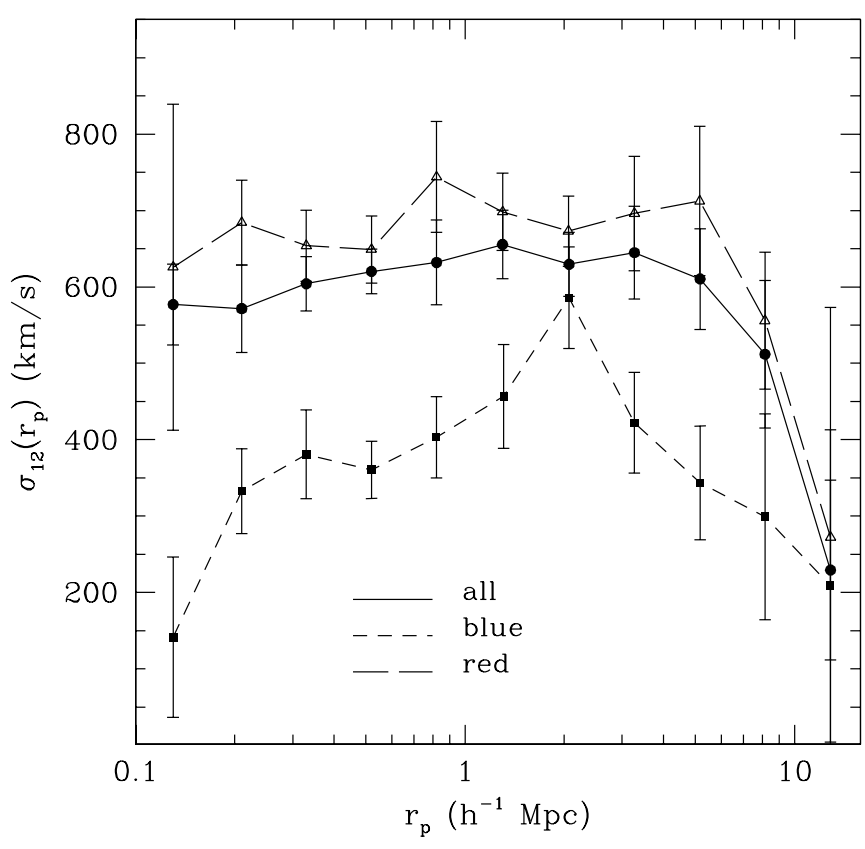

FIG. 15.-Pairwise velocity dispersion $\sigma_{12}\left(r_{p}\right)$ for the blue (short-dashed line), red (long-dashed line), and full (solid line) samples. All error bars are $1 \sigma$, derived from jackknife subsamples of the indicated galaxy class.

ence we obtain for the blue sample agree as well with a similar calculation by Jing, Börner, \& Suto (2002) using the PSCz survey. Our two subsamples have similar $\sigma_{12}$ at $r_{p}=15 h^{-1} \mathrm{Mpc}$; partial convergence of $\sigma_{12}$ at large scales is expected in theoretical models (Sheth et al. 2001a), though the assumptions used to infer $\sigma_{12}$ from $\xi\left(r_{p}, \pi\right)$ may also be breaking down at this point (see $\S 4.4$ and Fisher 1995). Calculations where $\bar{v}_{12}$ is assumed to be the same as that for dark matter particles in the $\Lambda \mathrm{CDM}$ model show again that the PVD at $r_{p} \lesssim 3 h^{-1} \mathrm{Mpc}$ are quite robust against the change of infall model.

\subsection{Luminosity}

We study the dependence of clustering on luminosity using three volume-limited subsamples, each with different absolute magnitude and redshift limits, as summarized in Table 3. The absolute magnitude ranges of the three subsamples are centered approximately on $M_{*}+1.5, M_{*}$, and $M_{*}-1.5$, where $M_{*}=-20.8$ is the characteristic luminosity in a Schechter (1976) function fit to the SDSS luminosity function (Blanton et al. 2001a; the other parameters in the fit are $\alpha=-1.2$ and $\phi_{*}=1.46 \times 10^{-2} h^{3} \mathrm{Mpc}^{-3}$ ). The space density of the lowest luminosity subsample is 27 times that of the highest luminosity subsample. The redshift ranges are chosen to ensure that the selection function $\phi(z)=1$ for each subsample (i.e., they are volume-limited), accounting for $K$-corrections and the bright and faint apparent magnitude limits of the full sample. Because the width of the absolute magnitude bins is half the range of apparent magnitudes in the full sample, the three redshift ranges are actually disjoint, so our comparison of clustering properties relies on each subsample volume being large enough to fairly represent the cosmic mean.

Figure 16 shows the projected correlation functions $w_{p}\left(r_{p}\right)$ for the three absolute magnitude subsamples. Table 3 lists the parameters $r_{0}$ and $\gamma$ of power-law $\xi_{r}(r)$ models

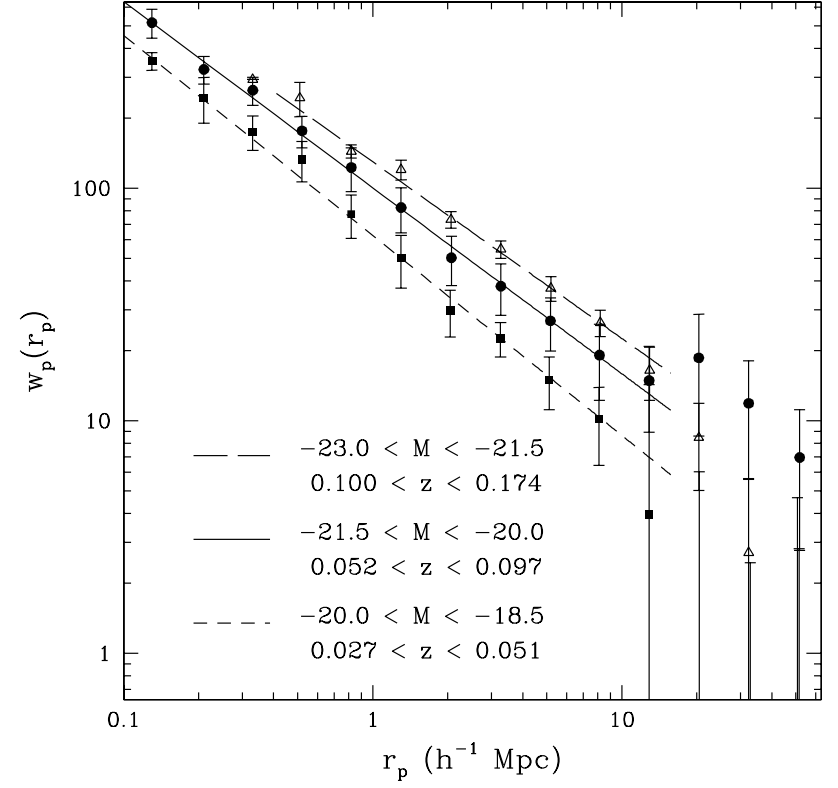

FIG. 16.-Projected correlation function $w_{p}\left(r_{p}\right)$ for three volume-limited samples, with absolute magnitude and redshift ranges as indicated. Squares, circles, and triangles show results for faint (sub- $M_{*}$ ), intermediate $\left(M_{*}\right)$, and luminous (super- $M_{*}$ ) galaxies, respectively. Short-dashed, solid, and long-dashed lines show the best-fit power-law models in the range they were fitted; parameters of the corresponding real-space $\xi_{r}(r)$ appear in Table 3.

determined by fitting $w_{p}\left(r_{p}\right)$ in the range $0.4 h^{-1} \mathrm{Mpc}<r_{p}<16 h^{-1} \mathrm{Mpc}$ for the highest luminosity subsample and $0.1 h^{-1} \mathrm{Mpc}<r_{p}<16 h^{-1} \mathrm{Mpc}$ for the other two subsamples. The correlation length and slope of the middle sample is similar to that of the full sample analyzed in $\S 4$, which is not surprising since most of the galaxies in a flux-limited sample have absolute magnitudes in the neighborhood of $M_{*}$. The low-luminosity subsample has a clustering amplitude that is lower by $\sim 40 \%$, and the highluminosity subsample has a clustering amplitude higher by $\sim 35 \%$.

The general trend of Figure 16, stronger clustering for more luminous galaxies, is similar to that found in a number of earlier studies (Davis et al. 1988; Hamilton 1988; White, Tully, \& Davis 1988; Park et al. 1994; Loveday et al. 1995; Guzzo et al. 1997; Benoist et al. 1998; Willmer et al. 1998; Norberg et al. 2001). However, while some of these studies (including Norberg et al.'s analysis of a large sample from the 2dFGRS) found that luminosity dependence became strong only for galaxies brighter than $M_{*}$, we find a steady trend from $M_{*}+1.5$ to $M_{*}-1.5$. Most of the earlier studies were based on $B$-band luminosities, while we have used $r^{*}$-band luminosities, and since clustering is color-dependent, this difference may partly or fully explain the difference in trend (see also Shepherd et al. 2001). As the SDSS sample grows, we will be able to examine luminosity dependence of clustering in greater detail over a wider dynamic range.

Perhaps the most remarkable aspect of Figure 16 is the nearly identical shape of the three correlation functions, i.e., "scale-independent luminosity bias"; at the $1 \sigma$ level each is consistent with a power-law $\xi_{r}(r)$ of slope $\gamma=1.8$ (see Table 3 ). The 2dFGRS analysis (Norberg et al. 2001) also recovers nearly identical power-law slopes in the different luminosity ranges. A "halo occupation" analysis of galaxy clustering 
TABLE 3

Volume-limited Correlation FunCtion Samples

\begin{tabular}{|c|c|c|c|c|c|c|}
\hline Absolute Magnitude Limits & Redshift Limits & $N_{\text {gal }}$ & $\bar{n}$ & $r_{0}$ & $\gamma$ & $r_{r_{0} \gamma}$ \\
\hline$-23.0<M_{r^{*}}<-21.5 \ldots \ldots \ldots \ldots$ & $0.100<z<0.174$ & 3,674 & 0.06 & $7.42 \pm 0.33$ & $1.76 \pm 0.04$ & -0.85 \\
\hline$-21.5<M_{r^{*}}<-20.0 \ldots \ldots \ldots \ldots$ & $0.052<z<0.097$ & 9,067 & 0.73 & $6.28 \pm 0.77$ & $1.80 \pm 0.09$ & -0.77 \\
\hline$-20.0<M_{r^{*}}<-18.5 \ldots \ldots \ldots \ldots$ & $0.027<z<0.051$ & 3,130 & 1.64 & $4.72 \pm 0.44$ & $1.86 \pm 0.06$ & -0.83 \\
\hline
\end{tabular}

Note.-All samples use $14.5<r^{*}<17.6 ; \bar{n}$ is measured in units of $10^{-2} h^{3} \mathrm{Mpc}^{-3} ; r_{0}$ is in units of $h^{-1} \mathrm{Mpc} ; r_{0}$ and $\gamma$ are obtained from a fit for $w_{p}\left(r_{p}\right)$; and $r_{r_{0}}$ is the normalized correlation coefficient between $r_{0}$ and $\gamma$.

(see, e.g., Benson et al. 1999; Ma \& Fry 2000; Peacock \& Smith 2000; Seljak 2000; Berlind \& Weinberg 2001; Scoccimarro et al. 2001) implies that the correlation function at submegaparsec scales is dominated by pairs of galaxies that reside in the same virialized dark halo, while the correlation function at scales $\gtrsim 2 h^{-1} \mathrm{Mpc}$ comes from pairs in separate halos. Maintaining the constant slope seen in Figure 16 requires maintaining the relative strength of these two contributions in galaxy populations that differ by a factor of 27 in space density and a factor of 2.3 in correlation amplitude, a delicate balancing act. This empirical result should prove a demanding constraint for theoretical models of galaxy formation.

\subsection{Dependence on Surface Brightness and Morphology}

The SDSS photometric pipeline (Lupton et al. 2001; R. H. Lupton et al., in preparation) measures many other properties that can be used to define galaxy classes. Here we consider two of these properties, surface brightness and light-profile concentration. Contrary to the case of the $u^{*}-r^{*}$ color cut, where the

bimodal distribution provides a natural place to divide the sample, for both these properties there is not an obvious place to cut, so our division is somewhat arbitrary in the middle of the distribution. Table 2 summarizes the thresholds that we use to define surface brightness and concentration subsamples, along with sample sizes, mean space densities, and correlation function parameters.

The surface brightness subsamples are divided at the threshold $\mu_{1 / 2}=20.5 \mathrm{mag} \operatorname{arcsec}^{-2}$, where $\mu_{1 / 2}=m$ $+2.5 \log _{10}\left(2 \pi r_{50}^{2}\right)$ is the mean $r^{*}$ surface brightness within the Petrosian half-light radius $r_{50}, K$-corrected and corrected for cosmological surface brightness dimming. The low surface brightness sample contains around 11,400 objects, and the high surface brightness sample contains around 17,900 objects. The left-hand panel of Figure 17 shows the projected correlation functions $w_{p}\left(r_{p}\right)$ of the two subsamples and of the full sample. The high surface brightness galaxies have a steeper $w_{p}\left(r_{p}\right)$ and a higher clustering amplitude at $r_{p} \lesssim 3 h^{-1} \mathrm{Mpc}$. Fits of a power-law $\xi_{r}(r)$ to points with $r_{p}<16 h^{-1} \mathrm{Mpc}$ yield $r_{0}=5.55 \pm 0.21 h^{-1}$ Mpc, $\gamma=1.55 \pm 0.04$ for the low surface brightness sample and $r_{0}=6.48 \pm 0.21 h^{-1} \mathrm{Mpc}, \gamma=1.84 \pm 0.03$ for the high surface brightness sample. This trend of clustering strength with surface brightness is consistent with some earlier results based on smaller samples (Bothun et al. 1993; Mo, McGaugh, \& Bothun 1994). The two correlation functions actually cross at large scales, contrary to the expectation from simple bias models (see Narayanan et al. 2000), but the $w_{p}\left(r_{p}\right)$ amplitudes on these scales are consistent with each other at the $1 \sigma$ level. We also note that since $w_{p}\left(r_{p}\right)$ is an integral in the $\pi$ direction out to $40 h^{-1} \mathrm{Mpc}$, its value at $r_{p}$ in fact probes clustering out to considerably larger scales.
The profile concentration subsamples are defined using the concentration parameter $c \equiv r_{90} / r_{50}$, which serves as a proxy for the traditional division of galaxies into early and late morphological types. For example, a pure de Vaucouleurs profile has $c \approx 3.3$ (given our definition of Petrosian magnitudes; see Blanton et al. 2001a), while a pure exponential profile has $c \approx 2.3$. We divide our full galaxy sample roughly in between these two values at $c=2.7$, yielding about 11,900 galaxies with high concentration and 17,400 galaxies with low concentration. The right panel of Figure 17 shows the projected correlation functions of these subsamples. As expected from earlier studies of morphologydependent clustering (e.g., Guzzo et al. 1997 and references therein), high- $c$ (early type) galaxies have a steeper, higher amplitude correlation function. Fits of a power law $\xi_{r}(r)$ yield $r_{0}=6.74 \pm 0.24 h^{-1} \mathrm{Mpc}, \gamma=1.88 \pm 0.02$ for the high-concentration subsample and $r_{0}=5.64 \pm 0.22 h^{-1}$ Mpc, $\gamma=1.63 \pm 0.03$ for the low-concentration subsample.

Qualitatively, our results for galaxy subsamples defined by surface brightness or profile concentration parallel our results for color subsamples described in $\S 5.1$. We have focused on $w_{p}\left(r_{p}\right)$ and $\xi_{r}(r)$, but the same characterization extends to redshift-space anisotropy: like red galaxies, high surface brightness and high concentration galaxies show strong fingers-of-God distortions, which in turn imply high PVDs. Given the well-known correlations between galaxy morphology, color, and surface brightness, these similarities are not surprising. As the SDSS progresses, it will be possible to extend this type of analysis to a much finer level; for example, seeing if surface brightness effects can be separated from color effects, isolating extreme classes of low surface brightness or compact galaxies, comparing the clustering of galaxies with high and low profile concentration at fixed

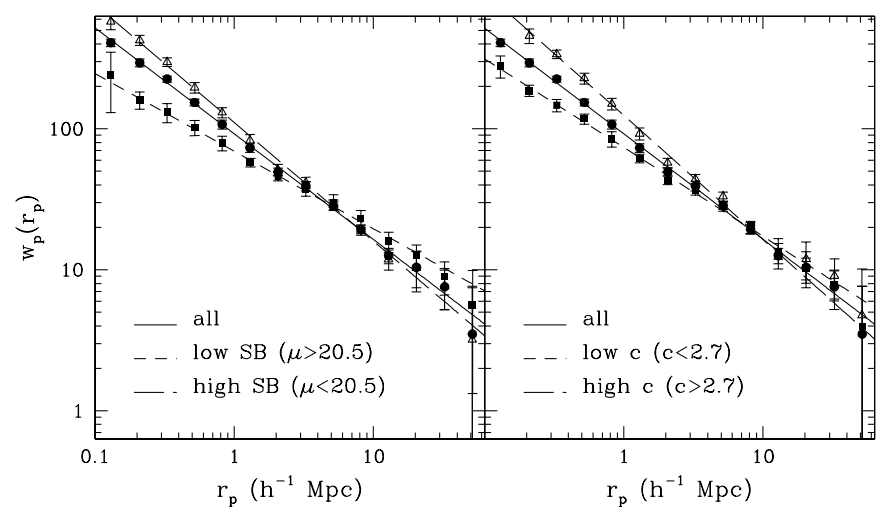

FIG. 17.-Projected correlation function $w_{p}\left(r_{p}\right)$ for samples cut according to (left) surface brightness $\mu$ and (right) concentration parameter $c \equiv r_{90} / r_{50}$. The straight lines correspond to the power-law fits of $w_{p}\left(r_{p}\right)$, for $r_{p}<16 h^{-1} \mathrm{Mpc}$. 
half-light surface brightness, or even comparing the clustering of barred and unbarred spirals or "disky" and "boxy" ellipticals. Detailed clustering studies of this sort should help disentangle the roles of early formation history and late-time transformation in determining galaxy properties.

\section{CONCLUSIONS}

We have presented the first measurements of galaxy clustering from early SDSS spectroscopic data, based on a sample of 29,300 galaxies. Since this sample covers a limited volume, spanning only $\sim 7 \%$ of the total projected survey area of the SDSS, our analysis has focused mainly on smallscale clustering. The sample used for this analysis has been chosen with care: in addition to a uniform flux limit imposed at bright and faint magnitudes, $14.5<r^{*}<17.6$, the sample is limited in radial velocity, $5,700 \mathrm{~km} \mathrm{~s}^{-1}<c z<39,000 \mathrm{~km} \mathrm{~s}^{-1}$, to avoid uncertainties introduced by evolution of the luminosity function, and in absolute magnitude, $-22<M_{r^{*}}-5 \log _{10} h<19$, so that it is dominated by galaxies with $r^{*}$-band luminosities around $M_{*}$. While these cuts reduce the number of galaxies included in the sample by nearly a factor of 2 , they allow robust conclusions to be drawn from the measurements. We have checked, for example, that our results are insensitive to details of the correlation function estimation, to uncertainties in the sample selection function, and to the effects of the $55^{\prime \prime}$ minimum fiber separation. As discussed in the Appendix below, we have also tested our jackknife error estimation method using a large number of mock redshift catalogs drawn from $N$-body simulations of cold dark matter models. These tests indicate that the jackknife errors used herein provide an accurate representation of the true statistical uncertainties over the scales of interest, at least in the context of these models.

For our full, flux-limited galaxy sample, we have measured the angle-averaged redshift-space correlation function $\xi(s)$ and the two-dimensional correlation function $\xi\left(r_{p}, \pi\right)$, projected the latter along the $\pi$ axis to infer the real-space correlation function $\xi_{r}(r)$, measured angular moments to quantify the anisotropy induced by peculiar motions, and modeled the small-scale anisotropy to infer the galaxy PVD $\sigma_{12}(r)$. Approximating the redshift-space correlation function by a power law, $\xi(s)=\left(s / s_{0}\right)^{-\gamma}$, yields a correlation length $s_{0} \approx 8 h^{-1} \mathrm{Mpc}$ and a slope $\gamma \approx 1.2$, but this representation is not accurate over a large range of scales. At small projected separations, contours of $\xi\left(r_{p}, \pi\right)$ show the characteristic fingers-of-God elongation along the line of sight caused by velocity dispersions in collapsed structures. At large separations, they show compression along the line of sight produced by coherent flows into high-density regions. The projected correlation function $w_{p}\left(r_{p}\right)$ can be well fitted by a power-law real-space correlation function $\xi_{r}(r)=\left(r / r_{0}\right)^{-\gamma}$, with $r_{0}=6.14 \pm 0.18 \quad h^{-1} \quad \mathrm{Mpc}$ and $\gamma=1.75 \pm 0.03$, for projected separations from 0.1 to 30 $h^{-1} \mathrm{Mpc}$. The ratio $Q(s)$ of the quadrupole and monopole moments of $\xi\left(r_{p}, \pi\right)$ is positive for $s \lesssim 10 h^{-1} \mathrm{Mpc}$, where fingers-of-God distortions dominate, and negative at larger scales, where coherent flow distortions dominate. A future analysis using a larger sample that extends to large scales in all three dimensions will enable us to extract an estimate of $\beta \equiv \Omega_{m}^{0.6} / b$ from the large-scale anisotropy. From the elongation of $\xi\left(r_{p}, \pi\right)$ at small scales, we estimate a PVD $\sigma_{12}(r) \approx 600 \mathrm{~km} \mathrm{~s}^{-1}$ that is roughly constant in the range $0.1 h^{-1} \mathrm{Mpc}<r<10 h^{-1} \mathrm{Mpc}$.

Our results for the full galaxy sample are in fairly good agreement with those obtained from earlier optically selected galaxy redshift surveys (see Table 1), in particular from clustering analyses of the LCRS (Tucker et al. 1997; Jing et al. 1998), which has similar selection, geometry, and size. The fact that our first analysis of early data from the SDSS reproduces these results and yields comparable or better statistical precision demonstrates the encouraging prospects for future galaxy clustering studies with the SDSS redshift survey. If we restrict our analysis to the subset of galaxies included in the SDSS Early Data Release (C. Stoughton et al. 2002, in preparation), we obtain very similar correlation function results, with larger statistical uncertainties because the sample is about half the size.

Taking advantage of our large sample and the high quality of SDSS imaging data, we have carried out a detailed examination of the dependence of real-space correlations and redshift-space distortions on galaxy photometric properties. Red and blue galaxies display markedly different clustering statistics, with the red galaxies exhibiting a higher amplitude and steeper real-space correlation function and much stronger fingers-of-God distortions than the blue galaxies; at $r_{p}=1 h^{-1} \mathrm{Mpc}$, the PVD is $\sim 750 \mathrm{~km} \mathrm{~s}^{-1}$ for our red galaxy subsample and $\sim 350 \mathrm{~km} \mathrm{~s}^{-1}$ for our blue galaxy subsample. Subsamples of high/low surface brightness and high/low profile concentration display qualitative behavior similar to that of the red/blue subsamples. Perhaps our most striking result is a measurement of luminosity bias of the real-space correlation function that is approximately scale-independent at $r \lesssim 10 h^{-1} \mathrm{Mpc}$. Using three volumelimited subsamples, we find a $\sim 40 \%$ decrease in clustering amplitude as we go from a median absolute magnitude of $M_{*}$ to $M_{*}+1.5$ and a similar increase when going from $M_{*}$ to $M_{*}-1.5$, implying relative biasing parameters $b / b_{*} \equiv\left(\xi / \xi_{*}\right)^{0.5}$ of 0.8 and 1.2 , respectively, for the faintest and brightest samples. These three samples differ by a factor of 27 in galaxy number density, but in each case $\xi_{r}(r)$ is consistent with a power law of slope $\gamma \approx 1.8$.

Studies of galaxy clustering and redshift-space distortions in the local universe have two main scientific objectives: (1) to test cosmological models and determine their parameters, and (2) to infer the relation between the galaxy and dark matter distributions, partly to sharpen cosmological tests, but mostly to constrain and guide the emerging theory of galaxy formation. Cosmological model tests usually focus on large scales, where the effects of nonlinear gravitational evolution and biased galaxy formation are relatively simple. These tests typically employ Fourier methods or statistical techniques that can isolate large-scale information and produce approximately uncorrelated error estimates even in the presence of a complicated survey geometry (Vogeley \& Szalay 1996; Tegmark, Taylor, \& Heavens 1997; Tegmark et al. 1998). Several of these methods have been applied to SDSS angular clustering data (Tegmark et al. 2002; Dodelson et al. 2002; Szalay et al. 2001), and they will be applied to the increasing sample of SDSS redshift data in the near future. The best constraints on galaxy bias will probably come from small and intermediate scales, where clustering statistics are most sensitive to the relation between galaxies and dark matter and where precise measurements can be obtained for many different classes of galaxies. Our results on the color and luminosity dependence of real-space clustering and on 
pairwise velocities already provide a challenging target for theories of galaxy formation. In the near future, these will be complemented by measurements of higher order clustering, which can break degeneracies among bias models that match two-point correlations (Scoccimarro et al. 2001; Szapudi et al. 2002). Studies of galaxy-galaxy weak lensing in the SDSS offer an entirely new route to determining the relation between galaxies and dark matter (Fischer et al. 2000; McKay et al. 2001). These measurements and other characterizations of galaxy clustering will improve in precision and detail as the SDSS progresses, yielding a wealth of new information with which to understand galaxy formation.

I. Z., M. R. B., and J. A. F. acknowledge support by the DOE and NASA grants NAG 5-7092 and NAG 5-10842 at Fermilab and by NSF grant PHY-0079251 at the University of Chicago. The Sloan Digital Sky Survey (SDSS) is a joint project of the University of Chicago, Fermilab, the Institute for Advanced Study, the Japan Participation Group, the Johns Hopkins University, the Max-Planck-Institute for Astronomy (MPIA), the Max-Planck-Institute for Astrophysics (MPA), New Mexico State University, Princeton University, the United States Naval Observatory, and the University of Washington. Apache Point Observatory, site of the SDSS telescopes, is operated by the Astrophysical Research Consortium (ARC). Funding for the project has been provided by the Alfred P. Sloan Foundation, the SDSS member institutions, the National Aeronautics and Space Administration, the National Science Foundation, the US Department of Energy, the Japanese Monbukagakusho, and the Max Planck Society. The SDSS World Wide Web site is http://www.sdss.org.

\section{APPENDIX}

\section{RELIABILITY OF ERROR ESTIMATES}

A complete model of galaxy clustering predicts, in addition to mean values, the distribution and covariance of statistical measurement errors for any specified sample geometry and selection function. These predicted statistical errors can be used to assess the consistency of the model with the data. However, such error estimates can be cumbersome to compute, and they depend on the assumed clustering model itself. For some purposes, therefore, it is desirable to have estimates of statistical errors and their covariances that depend only on the data set itself.

One common approach to this task is to estimate errors from disjoint subsamples of the full data set, each occupying a separate subvolume. One calculates the statistic of interest - e.g., $\xi\left(s_{i}\right)$ for a number of separations $s_{i}$-in each subvolume, and the estimated error of $\xi\left(s_{i}\right)$ is the error on the mean determined from the $N$ subvolumes. The same approach can be used to estimate covariance of errors. The disadvantage of this technique is that estimates of $\xi\left(s_{i}\right)$ from individual subvolumes may become noisy or biased, especially on scales comparable to the subvolume size. A related but more robust way to estimate errors from the sample itself is the jackknife method described in $\S 3.4$ (see specifically eq. [7]). In this approach, each jackknife subsample is obtained by excluding one of the subvolumes from the full sample, and one "sums up" the variances of the jackknife subsamples rather than taking the error of the mean. Since each jackknife subsample is similar in size to the full sample, this method performs better on large scales, though the two approaches should give equivalent results in the limit where each subvolume is representative of the whole data set (i.e., when fractional variances are small).

Our error bars on plotted data points and on parameter estimates $\left(r_{0}, \gamma, \sigma_{12}\right)$ are all computed using the jackknife method. Here we compare this approach to the model-based approach using the mock SDSS catalogs of Cole et al. (1998). Cole et al. (1998) ran a series of high-resolution $N$-body simulations, using an Adaptive $\mathrm{P}^{3} \mathrm{M}$ code (Couchman 1991), for a suite of cosmological models and biasing schemes. They created catalogs with the survey geometry and anticipated selection function of the SDSS. We use two of their catalogs: a $C O B E$-normalized flat $\Lambda$ CDM universe (with $\Omega_{m}=0.3$ and $h=0.65$ ) and a structure-normalized $\tau$ CDM model (with $\Omega_{m}=1.0, h=0.5$ and $\Gamma=0.25$ ). From each of these we extract 75 galaxy samples, each of which resembles the observed stripes in our sample. For computational convenience, we used artificial samples that are smaller than our current data set, with only $\sim 6,000$ galaxies per sample, but we expect that our conclusions about the relative behavior of jackknife and mock catalog error estimates would also hold for larger samples.

For each artificial galaxy sample we calculate the redshift-space $\xi(s)$ and the error estimates (including the full covariance matrix) using the jackknife method and the subvolume method. Figure 18 compares these estimated errors to the "true" errors of this model, defined as the scatter of the $75 \xi(s)$ estimates from different samples. Points and error bars show the mean and $1 \sigma$ scatter of the "internal " error estimates (jackknife or subvolume) in units of the true, "external" error at the same separation $s$.

The jackknife estimates recover the true errors reasonably well (with $1 \sigma$ scatter $\lesssim 50 \%$ ), and they are robust on all scales, without any gross systematics. The subvolume estimates do comparably well on the intermediate scales, but on large scales they overestimate the errors. The subvolume estimates are also more numerically unstable on small scales, where a single subvolume may contain few galaxy pairs.

The jackknife method produces unbiased estimates of the true errors for both cosmological models. These models are both designed to match the observed APM correlation function, so their predicted errors are also comparable, but they do differ by $\sim 20 \%$ on some scales, and the jackknife estimates seem to track these variations. We also performed this analysis for the offdiagonal elements of the covariance matrices. The covariance estimates do fairly well at intermediate scales but less well at small and large separations. The scatter in the estimates progressively increases for elements farther away from the diagonal. We are therefore less confident in the usefulness of the jackknife method for estimating a full covariance matrix; estimates of off-diagonal terms may be noisy and interdependent, making inversion of the covariance matrix unstable. When estimating parameters such as $r_{0}, \gamma$, and $\sigma_{12}$, therefore, we fitted values for each jackknife subsample using only diagonal terms, then estimated the error on the parameter by summing the variance of the estimates in the jackknife subsamples (see $\S \S 4.2$ and 4.4 ). This approach seems more reliable than using the full jackknife covariance matrix itself. 


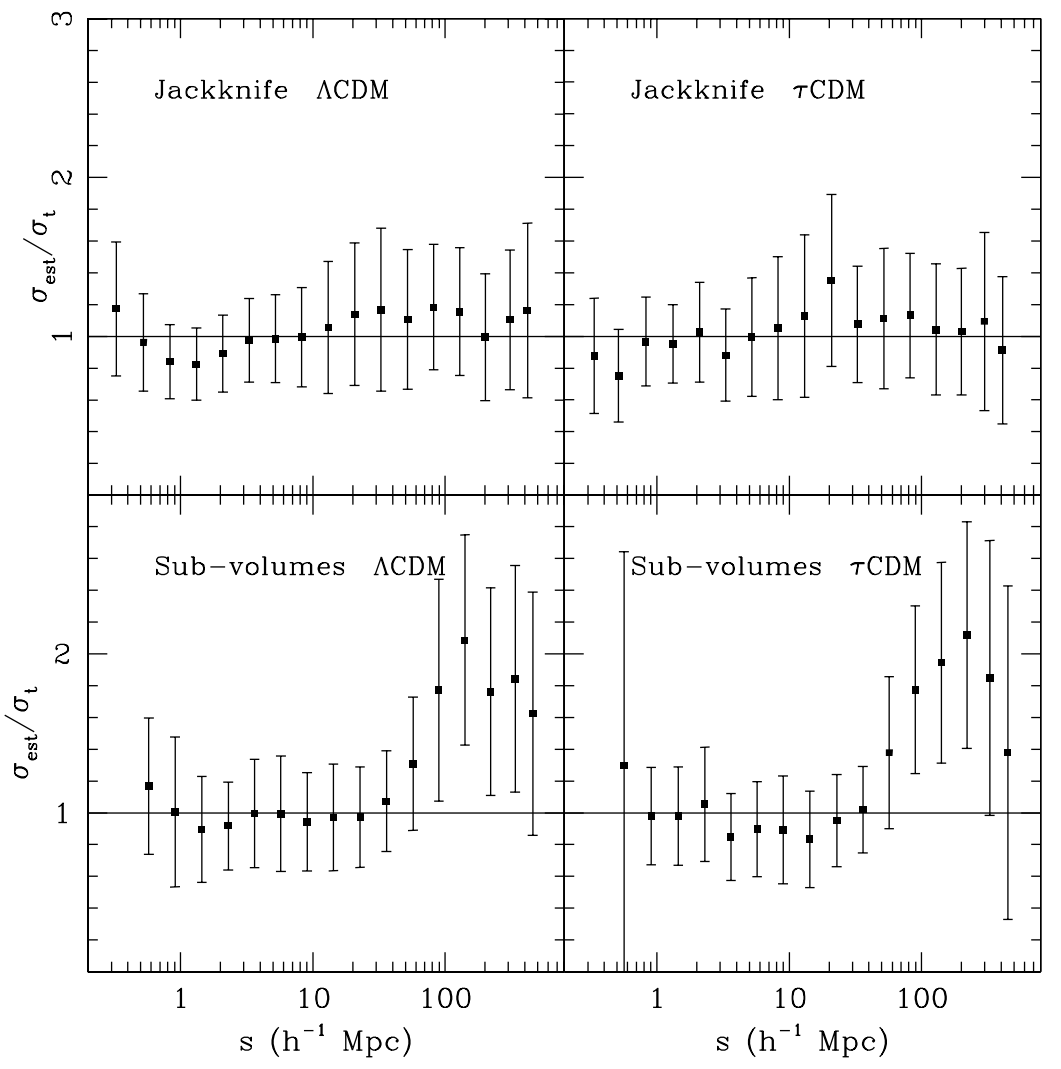

FIG. 18. - Comparison of jackknife and subvolume "internal" error estimates to "external" estimates derived from variation among artificial samples, using the $N$-body mock catalogs of Cole et al. (1998). Points and error bars show the mean and $1 \sigma$ scatter of the error estimates derived from 75 artificial samples, in units of the true (external) error. The top panels show results for the jackknife estimator, and bottom panels show results for the subvolume estimator. The left- and right-hand panels show $\Lambda \mathrm{CDM}$ and $\tau \mathrm{CDM}$ models, respectively.

For a specified clustering model, the mock catalog approach is probably the best way to assess the consistency of the model with the data, provided that one has an efficient way to create large numbers of mock catalogs of the necessary size. (Scoccimarro \& Sheth 2002 present a novel method that should improve the computational practicality of this approach for largescale surveys.) However, the tests presented here give us confidence that our jackknife error estimates should be representative of the true statistical error bars for models that have clustering similar to that observed. The jackknife approach is especially convenient when one breaks up the full sample into subsets that have different clustering properties, as we have done in $\S 5$, since it will automatically account for the influence of these clustering differences on the statistical errors. Scranton et al. (2001) have compared jackknife and mock catalog errors in the context of angular clustering measurements and reached similar conclusions.

Abell, G. O. 1958, ApJS, 50, 241

Bahcall, N. A., \& Soneira, R. 1983, ApJ, 270, 20

Bardeen, J., Bond, J. R., Kaiser, N., \& Szalay, A. S. 1986, ApJ, 304, 15

Bean, A. J., Ellis, R. S., Shanks, T., Efstathiou, G., \& Peterson, B. A. 1983, MNRAS, 205, 605

Benoist, C., Cappi, A., da Costa, L. N., Maurogordato, S., Bouchet, F. R., \& Schaeffer, R. 1998, ApJ, 514, 563

Benson, A. J., Cole, S., Frenk, C. S., Baugh, C. M., \& Lacey, C. G. 2000, MNRAS, 311, 793

Berlind, A. A., \& Weinberg, D. H. 2001, ApJ, submitted (astro-ph/ 0109001)

Blanton, M. R., Cen, R., Ostriker, J. P., \& Strauss, M. A. 1999, ApJ, 522, 590

Blanton, M. R., et al. 2001a, AJ, 121, 2358

Blanton, M. R., Lupton, R. H., Maley, F. M., Zehavi, I., \& Loveday, J. 2001b, AJ, submitted (astro-ph/0105535)

Bolzonella, M., Miralles, J.-M., Pelló, R. 2000, A\&A, 363, 476

Bothun G. D., Schombert J. M., Impey C. D., Sprayberry D., McGaugh S. S. 1993, AJ, 106, 548

Cen, R., \& Ostriker, J. P. 1992, ApJ, 399, L113

Cole, S., Fisher, K., \& Weinberg, D. H. 1994, MNRAS, 267, 785

Cole, S., Hatton, S., Weinberg, D. H., \& Frenk C. S. 1998, MNRAS, 300, 945

Coleman, G. D., Wu, C.-C., \& Weedman, D. W. 1980, ApJS, 43, 393

Coles, P. 1993, MNRAS, 262, 1065
REFERENCES

Colín, P., Klypin, A. A., Kravtsov, A. V., \& Khokhlov, A. M. 1999, ApJ, 523,32

Connolly, A. J., et al. 2001, ApJ, submitted (astro-ph/0107417)

Couchman, H. M. P. 1991, ApJ, 368, 23

Csabai, I., Connolly, A. J., Szalay, A. S., \& Budavari, T. 2000, AJ, 119, 69

da Costa, L. N., Pellegrini, P. S., Davis, M., Meiksin, A., Sargent, W. L. W., \& Tonry, J. L. 1991, ApJS, 75, 935

da Costa, L. N., et al. 1998, AJ, 116, 1

Davis, M., Efstathiou, G., Frenk, C. S., \& White, S. D. M. 1985, ApJ, 292, 371

Davis, M., \& Geller, M. J. 1976, ApJ, 208, 13

Davis, M., \& Huchra, J. P. 1982, ApJ, 254, 437

Davis, M., Meiksin, A., Strauss, M. A., da Costa, L. N., \& Yahil, A. 1988, ApJ, 333, L9

Davis, M., Miller, A., \& White, S. D. M. 1997, ApJ, 490, 63

Davis, M., \& Peebles, P. J. E. 1977, ApJS, 34, 425

. 1983, ApJ, 267, 465

de Lapparent, V., Geller, M. J., \& Huchra, J. P. 1986, ApJ, 302, L1 1986, ApJ, 332, 44

Diaferio, A., \& Geller, M. J. 1996, ApJ, 467, 19

Dodelson, S., et al. 2002, ApJ, in press (astro-ph/0107421)

Dressler, A. 1980, ApJ, 236, 351

Eisenstein, D., et al. 2001, AJ, 122, 2267

Falco, E. E., et al. 1999, PASP, 111, 438 
Feldman, H., Kaiser, N., \& Peacock, J. A. 1994, ApJ, 426, 23

Fischer, P., et al. 2000, AJ, 120, 1198

Fisher, K. B. 1995, ApJ, 448, 494

Fisher, K. B., Davis, M., Strauss, M. A., Yahil, A., \& Huchra, J. P. 1994, MNRAS, 267, 927

Fisher, K. B., Huchra, J. P., Strauss, M. A., Davis, M., Yahil, A., \& Schlegel, D. 1995, ApJS, 100, 69

Folkes, S. R., et al. 1999, MNRAS, 308, 459

Fry, J. N., \& Gaztañaga, E. 1993, ApJ, 413, 447

Fukugita, M., Ichikawa, T., Gunn, J. E., Doi, M., Shimasaku, K., \& Schneider, D. P. 1996, AJ, 111, 1748

Geller, M. J., et al. 1997, AJ, 114, 2205

Geller, M. J., \& Huchra, J. P. 1989, Science, 246, 897

Giovanelli, R., \& Haynes, M. P. 1985, AJ, 90, 2445

Gregory, S. A., \& Thompson, L. A. 1978, ApJ, 222, 784

Gunn, J. E., et al. 1998, AJ, 116, 3040

Guzzo, L., Strauss, M. A., Fisher, K. B., Giovanelli, R., \& Haynes, M. P. 1997, ApJ, 489, 37

Hamilton, A. J. S. 1988, ApJ, 331, L59

1992, ApJ, 385, L5

1993, ApJ, 417, 19

1998, The Evolving Universe (Dordrecht: Kluwer), 185

Hatton, S., \& Cole, S. 1998, MNRAS, 296, 10

Hermit, S., Santiago, B. X., Lahav, O., Strauss, M. A., Davis, M., Dressler, A., \& Huchra, J. P. 1996, MNRAS, 283, 709

Hogg, D. 1999, preprint (astro-ph/9905116)

Hubble, E. P. 1936, The Realm of the Nebulae ( Oxford: Oxford Univ. Press), 79

Huchra, J. P., Davis, M., Latham, D., \& Tonry, J. L. 1983, ApJS, 52, 89

Jing, Y.P., Börner, G., \& Suto, Y. 2002, ApJ, 564, 15

Jing, Y. P., Mo, H. J., \& Börner, G. 1998, ApJ, 494, 1

Joeveer, M., \& Einasto, J. 1978, in IAU Symp. 79, Large-Scale Structures in the Universe, ed. M. S. Longair \& J. Einasto (Dordrect: Reidel), 241

Juszkiewicz, R., Fisher, K. B., \& Szapudi, I. 1998, ApJ, 504, L1

Kaiser, N. 1984 ApJ, 294 , L9 1987, MNRAS, 227, 1

Katz, N., Hernquist, L., \& Weinberg, D. H. 1992, ApJ, 399, L109

Kauffmann, G., Colberg, J. M., Diaferio, A., \& White, S. D. M. 1999, MNRAS, 303, 188

Landy, S. D., \& Szalay, A. S. 1993, ApJ, 412, 64

Landy, S. D., Szalay, A. S., \& Broadhurst, T. J. 1998, ApJ, 494, L133

Lawrence, A., et al. 1999, MNRAS, 308, 897

Loveday, J., Maddox, S. J., Efstathiou, G., \& Peterson, B. A. 1995, ApJ, 442,457

Loveday, J., Peterson, B. A., Maddox, S. J., \& Efstathiou, G. 1996, ApJS, 107,201

Lupton, R. H. 1993, Statistics in Theory and Practice (Princeton: Princeton Univ. Press)

Lupton, R. H., Gunn, J. E., Ivezić, Z., Knapp, G. R., Kent, S., \& Yasuda, N. 2001, in ASP Conf. Ser. 238, Astronomical Data Analysis Software and Systems X, ed. F. R. Harnden, Jr., F. A. Primini, \& H. E. Payne (San Francisco: ASP), 269

Ma, C., \& Fry, J. N. 2000, ApJ, 543, 503

Mann, R. G., Peacock, J. A., \& Heavens, A. F. 1998, MNRAS, 293, 209

Marzke, R. O., Geller, M. J., da Costa, L. N., \& Huchra, J. P. 1995, AJ, 110,477

McKay, T., et al. 2001, AJ, submitted (astro-ph/0108013)

Melott, A. L., \& Fry, J. N. 1986, ApJ, 305, 1

Mo, H. J., Jing, Y. P., \& Börner, G. 1993, MNRAS, 264, 825 1997, MNRAS, 286, 979

Mo, H. J., McGaugh, S. S., \& Bothun G. D. 1994, MNRAS, 267, 129

Narayanan, V. K., Berlind, A. A., \& Weinberg, D. H. 2000, ApJ, 528, 1

Norberg, P., et al. 2001, MNRAS, 328, 64

Park, C., Vogeley, M. S., Geller, M. J., \& Huchra, J. P. 1994, ApJ, 431, 569

Peacock, J. A., et al. 2001, Nature, 410, 169
Peacock, J. A., \& Smith, R. E. 2000, MNRAS, 318, 1144

Pearce, F. R., et al. 1999, ApJ, 521, L99

Peebles, P. J. E. 1976, Ap\&SS, 45, 3

1980, The Large Scale Structure of the Universe (Princeton: Princeton Univ. Press)

Percival, W. J., et al. 2001, MNRAS, 327, 1297

Petrosian, V. 1976, ApJ, 209, L1

Ratcliffe, A., Shanks, T., Parker, Q. A., Broadbent, A., Watson, F. G., Oates, A. P., Collins, C. A., \& Fong, R. 1998, MNRAS, 300, 417

Regos, E., \& Geller, M. J. 1991, ApJ, 377, 14

Richards, G. T., et al. 2002, AJ, in press (astro-ph/0202251)

Santiago, B. X., Strauss, M. A., Lahav, O., Davis, M., Dressler, A., \& Huchra, J. P. 1995, ApJ, 446, 457

Sargent, W. L. W., \& Turner, E. L. 1977, ApJ, 212, L3

Saunders, W., et al. 2001, MNRAS, 317, 55

Schechter, P. 1976, ApJ, 203, 297

Scherrer, R. J., \& Weinberg, D. H. 1998, ApJ, 504, 607

Schlegel, D. J., Finkbeiner, D. P., \& Davis, M. 1998, ApJ, 500, 525

Scoccimarro, R., \& Sheth, R. K. 2002, MNRAS, 329, 629

Scoccimarro, R., Sheth, R. K., Hui, L., \& Jain, B. 2001, ApJ, 546, 20

Scranton, R., et al. 2001, ApJ, submitted (astro-ph/0107416)

Seljak, U. 2000, MNRAS, 318, 203

Shectman, S. A., Landy, S. D., Oemler, A., Tucker, D. L., Lin, H., Kirshner, R. P., \& Schechter, P. L. 1996, ApJ, 470, 172

Shepherd, C. W., Carlberg, R. G., Yee, H. K. C., Morris, S. L., Lin, H., Sawicki, M., Hall, P. B., \& Patton, D. R. 2001, ApJ, 560, 72

Sheth, R. 1996, MNRAS, 279, 1310

Sheth, R. K., Diaferio, A., Hui, L., \& Scoccimarro, R. 2001a, MNRAS, 326,463

Sheth, R. K., Hui, L., Diaferio, A., \& Scoccimarro, R. 2001b, MNRAS, 325,1288

Siegmund, W., et al. 2002, in preparation

Smith, J. A., et al. 2002, AJ, in press (astro-ph/02011437)

Somerville, R. S., Davis, M. \& Primack, J. R. 1997, ApJ, 479, 616

Stoughton, C., et al. 2002, AJ, in press

Strateva, I., et al. 2001, AJ, 122, 1861

Strauss, M. A., Huchra, J. P., Davis, M., Yahil, A., Fisher, K. B., \& Tonry, J. L. 1992, ApJS, 83, 29

Strauss, M. A., Ostriker, J., \& Cen, R. 1998, ApJ, 494, 20

Strauss, M. A., et al. 2002, AJ, submitted

Szalay, A. S., et al. 2001, ApJ, submitted, (astro-ph/0107419)

Szapudi, I., et al. 2002, ApJ, 570, 75

Tegmark, M., Hamilton, A., Strauss, M. A., Vogeley, M., \& Szalay, A. S. 1998, ApJ, 499, 555

Tegmark, M., Taylor, A. N., \& Heavens, A. F. 1997, ApJ, 480, 22

Tegmark, M., et al. 2002, ApJ, 571, 191

Tucker, D. L., et al. 1997, MNRAS, 285, L5

Uomoto, A., et al. 2002, in preparation

Vanden Berk, D. E., et al. 2002, in preparation

van de Weygaert R., \& van Kampen E. 1993, MNRAS, 263, 481

Vettolani, G., et al. 1998, A\&AS, 130, 323

Vogeley, M. S., \& Szalay, A. S. 1996, ApJ, 465, 34

White, M., Hernquist, L., \& Springel, V. 2001, ApJ, 550, L129

White, S. D. M., Tully, R. B., \& Davis, M. 1988, ApJ, 333, L45

Willmer, C. N. A., da Costa, L. N., \& Pellegrini, P. S. 1998, AJ, 115, 869

Yasuda, N., et al. 2001, AJ, 122, 1104

York, D. G., et al. 2000, AJ, 120, 1579

Yoshikawa, K., Taruya, A., Jing, Y. P., \& Suto, Y. 2001, ApJ, 558, 520

Zurek, W. H., Quinn, P. J., Salmon, J. K., \& Warren, M. S. 1994, ApJ, 431, 559

Zwicky, F. 1937, ApJ, 86, 217

Zwicky, F., Herzog, E., Wild, P., Karpowicz, M., \& Kowal, C. 1961-1968, Catalog of Galaxies and of Clusters of Galaxies, Vols. 1-6 (Pasadena: Caltech) 